\title{
A Comparison of Global Search Algorithms for Continuous Black-Box Optimization
}

Petr Pošík

posik@labe.felk.cvut.cz

Faculty of Electrical Eng., Czech Technical University in Prague, Czech Republic

Waltraud Huyer

Waltraud.Huyer@univie.ac.at

Faculty of Mathematics, University of Wien, Austria

László Pál

pallaszlo@sapientia.siculorum.ro

Faculty of Business and Humanities, Sapientia - Hungarian University of Transylvania, Miercurea-Ciuc, Romania

\begin{abstract}
Four methods for global numerical black-box optimization with the origins in mathematical programming community are described and experimentally compared with the state-of-the-art evolutionary method, BIPOP-CMA-ES. The methods chosen for the comparison exhibit various features potentially interesting for the evolutionary computation community: systematic sampling of the search space (DIRECT, MCS) possibly combined with a local search method (MCS), or a multistart approach (NEWUOA, GLOBAL) possibly equipped with a careful selection of points to run a local optimizer from (GLOBAL). The recently proposed "comparing continuous optimizers" (COCO) methodology was adopted as the basis for the comparison. Based on the results, we draw suggestions about which algorithm should be used depending on the available budget of function evaluations, and we propose several possibilities for hybridizing evolutionary algorithms with features of the other compared algorithms.
\end{abstract}

Keywords

Real-parameter optimization, continuous domain, black-box optimization, benchmarking, deterministic global optimization, stochastic global optimization.

\section{Introduction}

Global optimization is a subfield of applied numerical analysis which studies methods that should be able to find the globally optimal solution to an optimization problem. The issues and methods of global optimization are studied in several different communities. This article focuses on mathematical programming (MP) and evolutionary computation (EC) communities.

The ultimate goal of global black-box optimization-to make the fastest possible progress towards the best possible solution-is certainly common to both communities. There are, however, certain differences. The MP community strives for methods with sound theoretical properties. The methods often search the space systematically, build and use models of the objective function, and/or store all the points sampled during the run. Maintaining the models or using the archive of sampled points is very time and space-consuming. Due to practical limits in available CPU time and storage space, the MP community usually tests these algorithms using relatively small budgets of allowed function evaluations. As a result, the MP methods are designed to show a good 
progress right from the beginning of the search. The EC community studies algorithms with roots in nature and biology, often using randomly initialized populations. An EC algorithm needs some time to move the population to a promising region of the search space, and its performance in the initial phases is usually not as good as it could be. On the other hand, EC methods usually do not use any complex model or solutionarchive maintanance procedures and can be tested with higher evaluations budgets. As a consequence of these differences, the findings based on experimental results in these communities are often contradicting. This contributes to the gap between these two communities, and despite the fact we can learn a lot from each other, it does not happen very often.

This article adds a brick to the bridge between the MP and EC communities. It (re-)introduces several MP algorithms to the EC community and by means of experimental comparison, it highlights the differences in them, identifies the suitable algorithms for various goals and situations, and finally points out the features that may be profitable for the members of the EC community, and vice versa.

The article focuses on three MP methods with features not widely known in the EC community. These are complemented with two reference algorithms which are not discussed in such a detail as the three main algorithms since the experiments were not performed by us (the authors of the paper) and we have thus only a limited experience with them. The first method chosen for the comparison is the DIRECT algorithm (Jones et al., 1993). It systematically samples points from the search space and does not contain any dedicated local search method. The second algorithm, MCS (Huyer and Neumaier, 1999), works on similar principles as DIRECT, but contains also a specialized local search procedure. Both algorithms were described as "good complete general purpose global optimization algorithms" by Neumaier (2004, sec. 9). The third algorithm, GLOBAL (Csendes, 1988), is a multistart method equipped with a filter trying to prevent starting a local search in the basin of attraction of an already known local optimum. To contrast the effect of this filter with a usual multistart method, the restarted version of the NEWUOA algorithm (Powell, 2006) was also included in the comparison. It is a local optimizer proposed quite recently, but its reported results are promising. The final algorithm is the BIPOP-CMA-ES by Hansen (2009), a restarted version of the state-of-the-art CMA-ES algorithm using different population sizes in individual restarts. It represents the only evolutionary approach in the comparison and serves as the baseline algorithm. All the methods are described in Sec. 2.

A suitable experimental framework must be chosen to discover the potentially profitable features of the algorithms. The framework must be able to show the differences among the algorithms at all stages of the search, not just after certain number of evaluations, as is the usual practice. The COCO (Comparing Continuous Optimizers) methodology (Hansen et al., 2009a) was chosen since it fulfills these requirements. It was used as the basis of the Black-Box Optimization Benchmarking (BBOB) workshops of the GECCO 2009 and 2010 conferences. The testbed consists of 24 carefully chosen scalable noiseless benchmark functions (Hansen et al., 2009b) which represent various types of difficulties observed in real-world problems (ill-conditioning, multimodality, etc.). The COCO experimental framework is described in Sec. 3.

The results of the algorithms were already separately presented as the workshop papers (Hansen, 2009; Pošík, 2009; Ros, 2009b), or as unpublished reports (Huyer and Neumaier, 2009; Pál et al., 2009). One of the original contributions of this article is to collect these results, compare them conveniently in one place, and provide a discussion of the pros and cons of the algorithms compared to each other. In the 
original papers, the discussion (if any) was based solely on the results of the respective algorithm and no comparison was made. We also discuss the results in more detail than the summary paper of Hansen et al. (2010).

The setup of the experiments and the algorithm settings are described in Sec 4 . The results of the comparison are presented in Sec. 5. Sections 6 and 7 contain the discussion of the results broken down by the function group and by the algorithm, respectively. Sec. 8 summarizes the article and suggests several possible ways of using some of the MP principles to improve the evolutionary algorithms.

\section{The Compared Algorithms}

All the described algorithms are iterative. They sequentially sample points from the real-valued search space $X \subset \mathcal{R}^{D}$, where $D$ is the search space dimensionality. It is assumed hereafter that the points are evaluated as soon as they are sampled, and that the variables holding the best point found so far, $\mathrm{x}^{\text {best }}$, its function value, $f_{\text {best }}$, and the number of objective function evaluations are updated accordingly.

\subsection{DIRECT}

The DIRECT algorithm was introduced by Jones et al. (1993). The algorithm name not only expresses that it belongs to the class of direct search algorithms, it also describes the main principle of the algorithm: the DIRECT acronym stands for DIviding RECTangles. A slightly modified MATLAB implementation of DIRECT by Finkel (2003) is used. Only the basic algorithm design principles are described here; for the detailed description, see the original article by Jones et al. (1993) or the implementation description by Finkel (2003). The pseudocode of the algorithm is shown as Alg. 1.

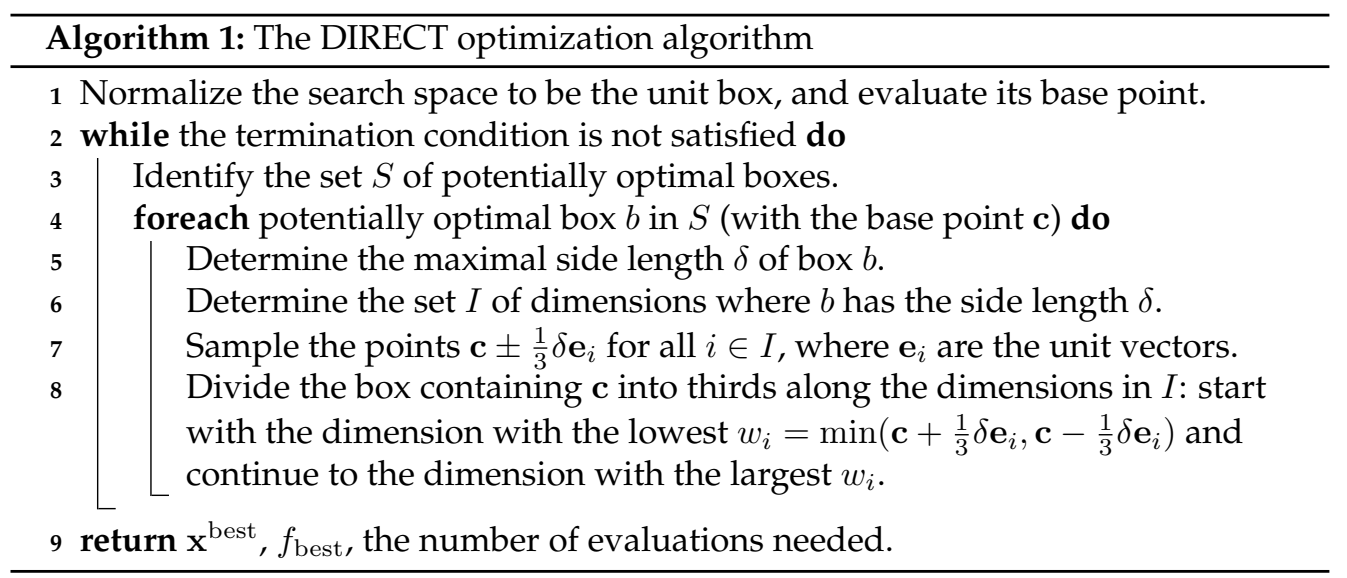

The algorithm is a branching scheme which recursively divides the search space and forms a tree of hyperrectangles (boxes). The leaves of the tree form a set of nonoverlapping boxes; in each time instant, the whole search space is completely covered by all the leaves. The point $\mathbf{c}$ in the middle of each box-the base point-is evaluated. Each box thus has two important characteristics: (1) the function value of its base point, and (2) the size of the box. There are many possible definitions of the box size, here the distance from the basepoint to the box corner is used.

In each iteration the algorithm decides which of the existing boxes should be split (see Alg. 1, line 3). The potentially optimal boxes are identified using 2 design princi- 
ples. It is expected that the chance of finding an improvement inside a box is proportional to

- the fitness of the base point (exploitation), and to

- the box size (exploration, global search).

The identification of the potentially optimal boxes is thus basically a multi-objective problem. Each iteration, all the non-dominated boxes described by their size and their base point function value are divided by the algorithm (see Alg. 1, lines 5-8). The division of boxes which are not potentially optimal, i.e. small boxes and boxes with worse base points, is thus postponed to the following iterations.

The DIRECT algorithm does not contain any local search method which could be used to improve its efficiency. The algorithm is guaranteed to eventually sample a point arbitrarily close to the global optimum, if it is allowed to run sufficiently long and if the splitting procedure is not constrained by a maximal depth.

\subsection{MCS}

Inspired by DIRECT, the global optimization algorithm multilevel coordinate search (MCS) was developed by Huyer and Neumaier (1999) to minimize an objective function on a box $[\mathbf{u}, \mathbf{v}]$ with finite or infinite bounds. The algorithm proceeds by splitting the search space into smaller boxes, but the splitting procedure is much more irregular than the one in DIRECT. By starting a local search from certain good points, an improved result is obtained. The pseudocode of the basic steps of MCS can be found in Alg. 2. The implementation used for the experiments can be downloaded from

http://www.mat.univie.ac.at/ neum/software/mcs/.

Each box in the partitioning process is characterized by (1) its bounds, (2) its "base point", and by (3) its level $s \in\left\{1, \ldots, s_{\max }\right\}$. The function is evaluated at the base points. They may lie on the box boundary and therefore the same base point can be shared by two or more boxes. The level of a box is a rough measure of the number of times the box has been processed. Like DIRECT, the MCS algorithm combines exploration (splitting boxes with a large unexplored territory) and exploitation (splitting boxes with good function values). Boxes with the level $s_{\max }$ are considered too small for further splitting. Whenever a box with the level $s<s_{\max }$ is split, its descendants get the level $s+1$ or $s+2$. At each stage of the algorithm, the partitioning of the search space consists of a set of boxes with levels between 1 and $s_{\max }$.

The algorithm starts with the so-called initialization procedure (lines 2-5 of Alg. 2). For each coordinate $i=1, \ldots, D$, at least three values $x_{i}^{1}<x_{i}^{2}<\cdots<x_{i}^{L_{i}}$ in $\left[u_{i}, v_{i}\right]$ are needed, where $L_{i} \geq 3$ and the coordinates $x_{i}^{0}$ of the initial point belong to $\left\{x_{i}^{1}, \ldots, x_{i}^{L_{i}}\right\}$. These values are used whenever a box is split in the coordinate $i$ for the first time (in the initialization procedure or later). Splits are made (at the values of the initialization list and between) into at least $2\left(L_{i}-1\right) \geq 4$ parts along each coordinate $i=1, \ldots, D$.

The main iteration loop (lines 6-20 of Alg. 2) proceeds (in the absence of other stopping criteria) until all boxes of the current partitioning have the level $s_{\max }$. Additional stopping criteria like reaching a target function value or a limit on the number of function evaluations are implemented but not shown in the pseudocode. In each iteration, the algorithm splits one box at each level, starting with the smallest non-empty level (i.e., with the largest boxes). When a box with the base point $\mathbf{x}$ is split, that is done along a single coordinate $i$ and the function is evaluated at one or more points differing from $\mathrm{x}$ only in the coordinate $i$. 


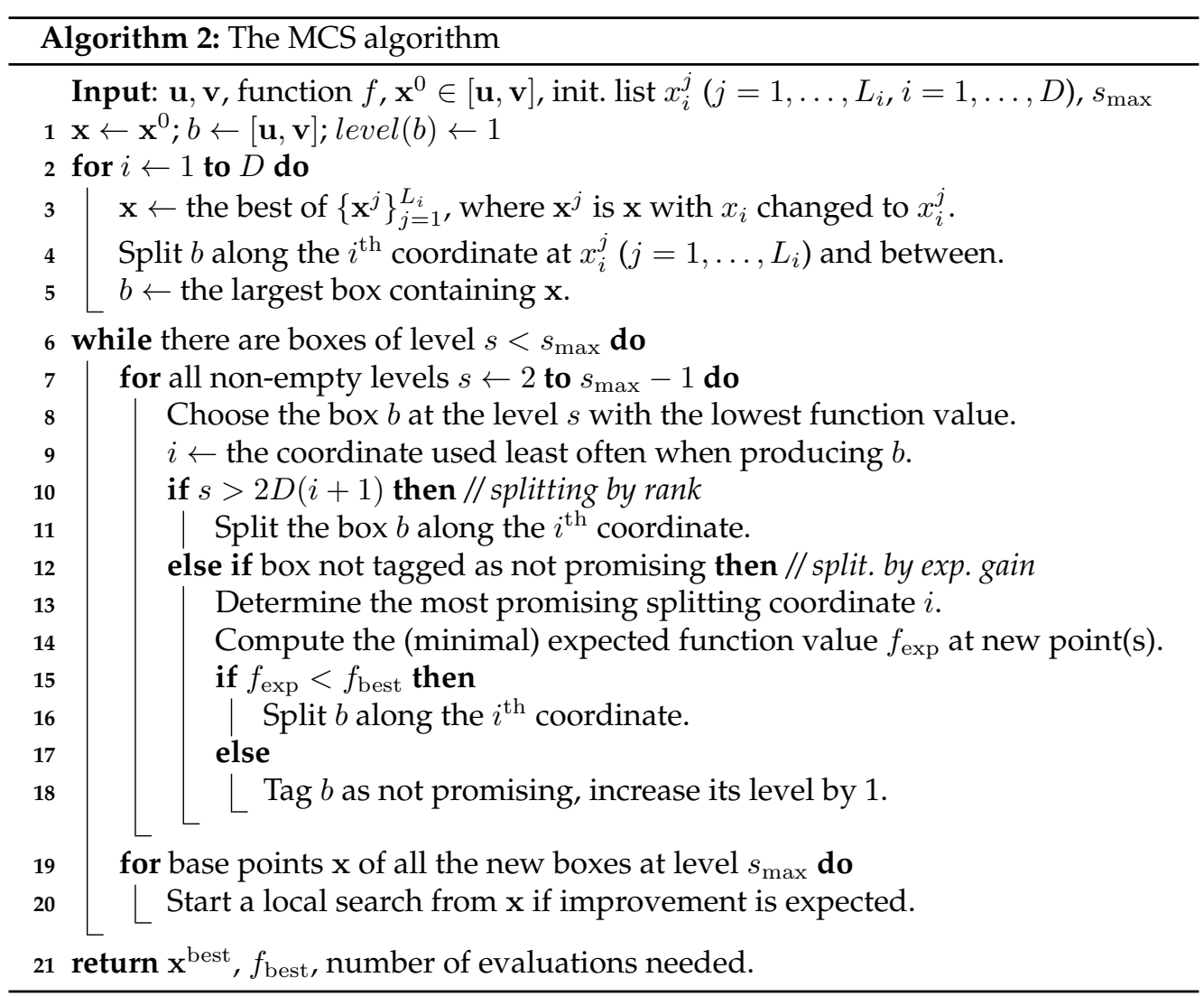

To split a box at the level $s$ with the base point $\mathrm{x}$ and the bounds $\underline{\mathrm{x}}$ and $\overline{\mathrm{x}}$, the algorithm has to choose the splitting dimension $i$ and the position of the split (based on information gained from already sampled points). Two kinds of splits can occur:

1. Splitting by rank: If a box has already reached a high level but still hasn't been split very often in some coordinate $i$, the function is evaluated at a point obtained by changing the $i$ th coordinate of $\mathrm{x}$ to a value depending on $x_{i}, \underline{x}_{i}$ and $\bar{x}_{i}$, and the box is split into three parts.

2. Splitting by expected gain: Otherwise, the splitting coordinate $i$ and the $i$ th coordinate of the new point are determined by building a separable local quadratic model around $\mathrm{x}$ and minimizing it, with safeguards to prevent too narrow splits. Two or three subboxes are obtained.

In both cases, the given recipes only apply to the case that the box has already been split along the coordinate $i$. If that is not the case, the function is evaluated at the points obtained by changing $x_{i}$ to the other values of the initialization list. The splits between two points where the function has been evaluated (according to the initialization list or otherwise) are not made symmetrically: the part with the lower function value gets the larger space. The larger parts of splits get the level $s+1$ and the smaller parts get the level $\min \left(s+2, s_{\max }\right)$.

MCS with local search (line 20) tries to accelerate the convergence of the algorithm by starting local searches from the points belonging to boxes of level $s_{\max }$. 
The local search algorithm essentially consists of building a local quadratic model by triple searches, then defining a promising search direction by minimizing the quadratic model on a suitable box and finally making a line search along this direction. This is repeated until the maximal number of iterations $n s_{\text {loc }}$ for the local search algorithm is reached, the algorithm does not make any progress any more, or the estimated gradient becomes too small (unless one of the stopping criteria used for MCS is satisfied first).

If the number of levels $s_{\max }$ goes to infinity, MCS is guaranteed to converge to the globally optimal function value if the objective function is continuous in the neighborhood of a global optimizer. This follows from the fact that then the set of points sampled by MCS is a dense subset of the search space.

\subsection{GLOBAL}

The stochastic global optimization method called GLOBAL (Csendes, 1988) was inspired by Boender et al. (1982) and was developed to solve bound constrained global optimization problems with black-box type objective functions. The goal of GLOBAL is to find all local minima that are potentially global. For this purpose it is equipped with a multistart strategy and clustering to promote finding distinct local optima.

Based on the old GLOBAL method (Csendes, 1988), after a careful study a new version (Csendes et al., 2008) was developed achieving better reliability and efficiency while allowing higher dimensional problems to be solved. In the new version we use the quasi-Newton local search method with the BFGS update instead of the earlier DFP. The algorithm implementation is available for academic and nonprofit purposes at

http: //www. inf.u-szeged.hu/ csendes/Reg/regform.php.

The main steps of GLOBAL are summarized in Alg. 3. As a multistart method, GLOBAL iteratively samples new points from the search space $X$ according to the uniform distribution (global phase, line 3 of Alg. 3), and executes a local search procedure starting from some of those points (local phase, line 8). The GLOBAL differs from the other multistart methods in two important aspects:

- Not all the points sampled during the global phase (the cumulated sample $S_{C}$ ) are considered as good candidates for starting the local search. Only the best $100 \gamma \%$ of them are used (the reduced sample $S_{R}$, line 4).

- These selected points are further filtered. The algorithm tries to prevent running a local search in the basin of attraction of an already detected local minimizer.

The filter is realized by a clustering procedure. The goal of the clustering procedure is to maintain one cluster per basin of attraction of a local optimum. GLOBAL uses the Single Linkage clustering rule (Boender et al., 1982; Rinnooy Kan and Timmer, 1987). The clusters are updated each iteration and only grow with time. A new point is added to the cluster if it is within a critical distance from a point already in the cluster initiated by a seed point. If the new point is not close enough to any already clustered point, it remains unclustered and thus a candidate for the local search initiation. The seed points are the local optima found so far, i.e. the members of the $X^{*}$ set. The distribution of all the clustered points approximates the level set of the function; each connected component of the level set (each cluster) then approximates the shape of one basin of attraction initiated by its respective local optimum $\mathrm{x}^{*} \in X^{*}$.

The filtering (using clustering) is applied each iteration after the reduced set creation (line 4), and after the identification of a new seed point (line 12). In the first iteration, $X^{*}$ is empty and thus no clustering takes place. The applied critical distance depends on the total sample size $\left|S_{C}\right|$ and it is constructed in such a way that the prob- 
A Comparison of Global Search Algorithms

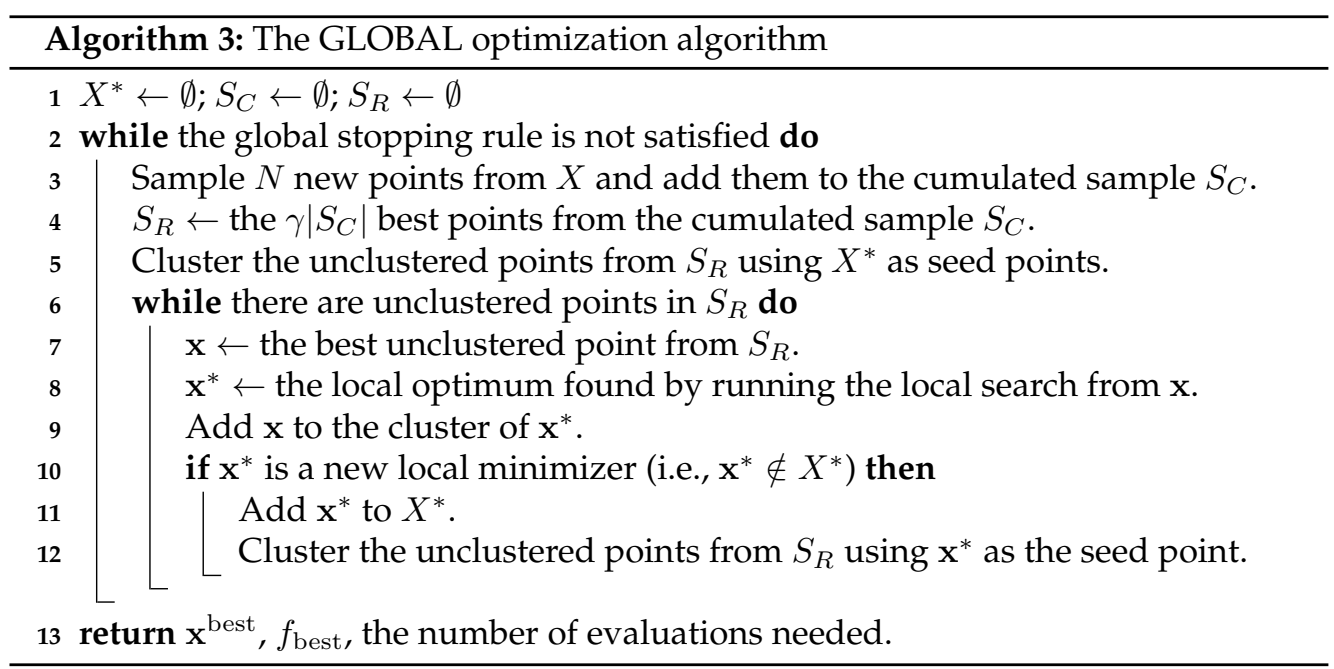

ability that the local method will be started tends to zero when the size of the sample grows (Boender et al., 1982; Rinnooy Kan and Timmer, 1987). The algorithm stops the search when it does not find any new local minimizer during the last iteration.

\subsection{Reference algorithms}

Two other optimization algorithms were selected as competitors for the just described global search algorithms: the NEWUOA procedure and the bi-population evolutionary strategy with covariance matrix adaptation (BIPOP-CMA-ES).

NEWUOA (Powell, 2006) was selected since it is a relatively recent optimization procedure with very promising reported results on various test functions. It is a deterministic (with the exception of initialization) local search procedure using quadratic modeling and a trust-region approach. The method maintains a quadratic model of the objective function in the trust region. Before each iteration, the model must interpolate the function at $m$ points, with $m$ typically equal to $2 D+1$, which is a much lower number of constraints than would be needed to specify a full quadratic model. The remaining degrees of freedom are taken up by minimizing the Frobenius norm of the difference between the new and the old quadratic model.

BIPOP-CMA-ES (Hansen, 2009) was chosen since it was one of the best algorithms in the BBOB-2009 comparison regarding the proportion of functions solved (Hansen et al., 2010). It is a multistart strategy using the original CMA-ES algorithm (with slightly modified parameter values) as the basic local search engine. The individual restarts differ only in the population size. Two strategies of population size setting are interlaced. The first strategy multiplies its population size by a factor of 2 each time it is executed. The second strategy chooses the population size randomly, somewhere between the initial minimal population size and the half of the last population size used by the first strategy. Increasing the population size slows down the algorithm convergence, on the other hand it results in a more global and robust search.

\section{Experimental Framework Description}

The experiments were carried out using the Comparing Continuous Optimizers (COCO) framework (Hansen et al., 2009a), which was also used as the basis for the 
Black-box Optimization Benchmarking workshop at the GECCO 2009 and 2010 conferences. The numerical experiments are performed on a testbed consisting of 24 noiseless test functions (Finck et al., 2009a; Hansen et al., 2009b). These functions reflect the real-world application difficulties and are categorized by function properties as multimodality, ill-conditioning, global structure and separability. The role of the categories is to reveal the different aspects of the algorithms. All functions are scalable with the dimension $D$ and their search domain is $[-5 ; 5]^{D}$. Each of the functions has 5 instances which differ in rotation and offset. The experiment shall be repeated three times for each instance, which means 15 trials for an algorithm on each function. Since DIRECT is a deterministic algorithm, only 1 trial of each instance was carried out.

An optimization problem is defined as a particular (function, requested target value) pair. Each function is used to define several optimization problems differing in the requested target value $f_{\mathrm{t}}=f_{\mathrm{opt}}+\Delta f_{\mathrm{t}}$, where $f_{\mathrm{opt}}$ is the optimal function value, and $\Delta f_{\mathrm{t}}$ is the precision (or tolerance) to reach. The success criterion of a trial (for each optimization problem) is to reach the requested target value $f_{\mathrm{t}}$. Many precision levels $\Delta f_{\mathrm{t}} \in\left[10^{-8}, 10^{2}\right]$ are defined. If the optimizer solves a function to the ultimate precision value $10^{-8}$, it actually solves many optimization problems along the way, and we shall say that it has found the optimum of the function. If the optimizer cannot reach the ultimate precision, it can gain some points for optimizing the function at least partially.

The main performance measure used in the COCO framework is the Expected Running Time, ERT (Hansen et al., 2009a; Price, 1997). The ERT estimates the expected number of function evaluations needed to reach the particular target function value if the algorithm is restarted until a single success. The ERT thus depends on the given target function value, $f_{\mathrm{t}}$, and is computed as "the number of function evaluations conducted in all trials, while the best function value was not smaller than $f_{\mathrm{t}}$ during the trial, divided by the number of trials that actually reached $f_{\mathrm{t}}$ " (Hansen et al., 2009a).

The results are presented using the Empirical Cumulative Distribution Function (ECDF). It shows the empirical cumulated probability of success on the considered problems depending on the allocated budget. The ECDF of the ERT is constructed as a bootstrap distribution of the ERT divided by the problem dimension $D$. In the bootstrapping process, 100 instances of ERT are generated by repeatedly drawing single trials with replacement until a successful trial is drawn for each optimization problem.

Since the ECDF graphs express the proportion of solved problems, rather than the reached function values, it is possible to meaningfully aggregate the ECDF graphs for several functions of the same class into one graph. The downside of this aggregation is that we are not able to distinguish the individual functions. In an ECDF graph aggregating the results of 5 functions, reaching the $20 \%$ level of solved problems after $n$ evaluations may mean many things. On the one hand, the algorithm could have found the minimum of one of the five functions, while the other functions may still remain completely unsolved. On the other hand, it may mean that only the problems related to the loose target levels were solved across all the aggregated functions. The latter case is the usual one. If the former explanation is the right one, we will point it out explicitly.

An additional measure used in COCO is the crafting effort (Price, 1997; Hoos and Stützle, 1998) that characterizes the parameter tuning effort for an algorithm. The crafting effort should be calculated for each dimension in the following way: $\mathrm{CrE}=-\sum_{k=1}^{K} \frac{n_{k}}{n} \ln \left(\frac{n_{k}}{n}\right)$, where $K$ is the number of different parameter settings, $n$ is the number of functions in the testbed, and each $n_{k}$ is the number of functions for which the $k$-th parameter setting was used. The CrE is zero in a given dimension $D$ if the setting was identical for all functions. 


\section{Algorithm and Experiment Parameter Settings}

The following subsections describe the experimental setup and the parameter settings of DIRECT, MCS, and GLOBAL. For the settings of the reference algorithms, we refer the reader to the original reports (Ros, 2009b; Hansen, 2009).

All experiments were run using the BBOB-2009 settings which required to benchmark all the algorithms in the dimensions $D=2,3,5,10,20$ and optionally in $D=40$. In this article, we do not consider the 40-D case since the 20-D space is already enough to show the main characteristics of the individual algorithms and to emphasize the differences among them.

\subsection{DIRECT}

The DIRECT algorithm was not restarted; a single run was carried out and stopped after reaching the final precision $\Delta f_{\mathrm{t}}=10^{-8}$ or after $10^{5}$ function evaluations.

The Jones factor $\epsilon$ is the minimal amount of improvement which is considered to be significant by the algorithm. The value was set to $\epsilon=10^{-10}$.

The maximal depth of the division tree was set to 21 . It is roughly equivalent to setting the minimal allowed distance between two neighbouring sampled points (under assumption that the division always takes places along the shortest box side, which is not true). With the maximal depth set to 21 , the theoretical minimal distance is of order $10^{-9}$, but it is larger in practice.

The initial bounding hypercube was set to $\langle-6,6\rangle^{D}$ despite the fact that all the benchmark functions have the global optimum in $\langle-5,5\rangle^{D}$ (Hansen et al., 2009a). Several of the benchmark functions have the global optimum near (or directly on) the search space boundary. Since DIRECT is pretty bad in approaching such solutions, the larger box was chosen. ${ }^{1}$

DIRECT is completely deterministic-only 1 run (instead of 3) for each function instance was carried out. The same parameter settings were used for all experiments on all functions, the crafting effort is $\mathrm{CrE}=0$ for all $D$.

\subsection{MCS}

MCS is equipped with meaningful default values for all parameters. We use the values $s_{\max }=5 D+10$ (default) for the number of levels, $n f_{\max }=500 \cdot \max (D, 10)$ for the limit on the overall number of function calls, $n s_{\text {loc }}=n f_{\max } / 5$ (much larger than the default value 50) for the limit on the number of iterations in a local search, and reaching the final precision $\Delta f_{\mathrm{t}}=10^{-8}$ as an additional stopping criterion.

The bounding box is given by $\mathbf{u}=(-5, \ldots,-5)^{T}$ and $\mathbf{v}=-\mathbf{u}$. The default MCS initialization list for finite $\mathbf{u}$ and $\mathbf{v}$ consists of the boundaries and the midpoint, with the midpoint as the starting point. The second initialization list for finite bounds uses $x_{i}^{1}=\frac{5}{6} u_{i}+\frac{1}{6} v_{i}, x_{i}^{2}=\frac{1}{2}\left(u_{i}+v_{i}\right), x_{i}^{3}=\frac{1}{6} u_{i}+\frac{5}{6} v_{i}, i=1, \ldots, D$, and again $\mathbf{x}^{0}=\frac{1}{2}(\mathbf{u}+\mathbf{v})$. The third option is to use global line searches along each coordinate, starting from the absolutely smallest point in the box, and generate at least three values for each coordinate. We call the MCS algorithm with these three kinds of initialization lists MCS1, MCS2, and MCS3, respectively. A user-defined initialization list is another option. After the initialization list has been chosen, MCS is purely deterministic.

In order to give MCS another chance to solve a problem in the case that the algorithm gets stuck in a nonglobal minimizer, and to introduce a random element in the

\footnotetext{
${ }^{1}$ It was observed that this choice improved the results e.g. for the linear slope function, while it did not worsen the results on the rest of the functions.
} 
algorithm at the same time (so that repeating the experiment three times for each instance becomes meaningful), we do not make a single call to MCS with a larger function evaluation budget but instead each experiment consists of up to 10 independent calls to MCS with the above parameters (i.e., each call to MCS does not use any results of the previous calls). First, MCS1, MCS2, and MCS3 are applied to the problem. Then initialization lists with the values $x_{i}^{1}<x_{i}^{2}<x_{i}^{3}$ drawn uniformly from $\left[u_{i}, v_{i}\right]$ for $i=1, \ldots, D$ and $x_{i}^{0}=x_{i}^{2}$ are used for at most 7 times for the dimensions $D=2,3,5$ and at most 5 times for the dimensions $D=10,20$ (in order to save CPU time).

Since the same parameter settings were used for all experiments on all functions, the crafting effort is $\mathrm{CrE}=0$ for all $D$.

\subsection{GLOBAL}

The COCO framework suggests to compare multistart versions of the base algorithms, i.e. to conduct independent restarts during each trial. However, the GLOBAL algorithm itself is a multistart procedure so that no restarts of GLOBAL were carried out.

GLOBAL has six parameters to set: the number $N$ of points to sample in each iteration, the proportion $\gamma$ of the best points selected for the reduced sample, the stopping criterion for the local search, the maximum number of function evaluations allowed for local search, the maximum number of local minima to be found (i.e. the maximum number of clusters to be maintained), and the type of the used local search method. All these parameters have a default value and usually it is enough to change only the first three of them.

In all dimensions and for all functions, we sampled $N=300$ new points ${ }^{2} ; \gamma=\frac{2}{N}$, so that the reduced sample contains less than $1 \%$ best points ever sampled.

The following settings were used for $D=2,3,5$. We used the Nelder-Mead simplex method (Nelder and Mead, 1965) implemented in MATLAB by Kelley (1999) as the local search procedure. The termination tolerance parameter TolFun was set to $10^{-8}$ and the maximum number of function evaluations was equal to 5,000 .

For $D=10,20$, two different settings were used. For the functions $f_{3}, f_{4}, f_{7}, f_{16}$, and $f_{23}$, we used the previous settings with the TolFun parameter set to $10^{-9}$. The reason for this choice was that the functions $f_{7}, f_{16}$, and $f_{23}$ are not smooth and the BFGS method performs poorer on them. On the functions $f_{3}$ and $f_{4}$ the simplex method performs slightly better. For the remaining functions, we used the MATLAB fminunc function as the local search method using the BFGS update formula with 10,000 as the maximum number of function evaluations and with TolFun set to $10^{-9}$. The meaning of the termination tolerance TolFun is different for each of the two local search methods. In case of the Nelder-Mead simplex method it is related to the diameter of the simplex, while in the case of BFGS it relates to the size of the gradient.

The crafting effort $\mathrm{CrE}=0$ for dimensions 2, 3, and 5. However, for $D=10,20$ two different setting were used. The crafting effort can be calculated as $\mathrm{CrE}_{10}=\mathrm{CrE}_{20}=$ $-\left(\frac{5}{24} \ln \frac{5}{24}+\frac{19}{24} \ln \frac{19}{24}\right)=0.5117$.

\section{Results}

Results from experiments according to Hansen et al. (2009a) on the benchmark functions (Finck et al., 2009b; Hansen et al., 2009b) are presented in Figures 1 and 2. Only the results for $D=5$ (exemplar of "low" dimensionality) and $D=20$ (exemplar of

\footnotetext{
2 The value was chosen after a preliminary testing. The default value was to small in case of many functions (with many local minimizers) in smaller dimensions. On the other hand, larger values did not improve the results in larger dimensions.
} 
"higher" dimensionality) are presented.

Tables 1 to 10 give the Expected Running Time (ERT) for the target precisions $10^{1,0,-1,-3,-5,-7}$ divided by the best ERT obtained during BBOB 2009 (given in the $\mathrm{ERT}_{\text {best }}$ row), together with a measure of its spread (the value typeset in parentheses with a smaller font gives the half of the range between the 10th and 90th percentile). Bold entries correspond to the 3 best values among the algorithms compared. The median number of conducted function evaluations is additionally given in italics, if $\operatorname{ERT}\left(10^{-7}\right)=\infty$. \#succ is the number of trials that reached the final target $f_{\text {opt }}+10^{-8}$.

The BIPOP-CMA-ES algorithm was used as the baseline for the statistical comparison of the other algorithms studied in this article. Each algorithm is tested if it improved the results obtained by BIPOP-CMA-ES. The statistical significance is tested with the rank-sum test for a given target $f_{\mathrm{t}}$ using, for each trial, either the number of needed function evaluations to reach $f_{\mathrm{t}}$ (inverted and multiplied by -1 ), or, if the target was not reached, the best $\Delta f$-value achieved, measured only up to the smallest number of overall function evaluations for any unsuccessful trial under consideration if available. Entries with the $\downarrow$ symbol are statistically significantly better (according to the rank-sum test) compared to the BIPOP-CMA-ES, with $p=0.05$ or $p=10^{-k}$ where $k>1$ is the number following the $\downarrow$ symbol, with Bonferroni correction of 24 .

\section{Discussion by Function Group}

In this section, the discussion of the results is broken down by function groups. The discussion mostly applies to the presented results for 5-D and 20-D. For a discussion on the individual algorithms, see Sec. 7.

\subsection{All functions aggregated}

The results for all functions are aggregated in the ECDF graphs of ERT for the 5-D and 20-D functions in Figs. 1 and 2, respectively, in the upper left part.

In the 5-D space, for very low budgets of function evaluations (\#FEs $<20 D$ ), NEWUOA and MCS are (close to) the best of all algorithms ever compared using the BBOB methodology. They stay the best among the algorithms compared in this article for $\# \mathrm{FEs}<200 D$. For $200 D<\# \mathrm{FEs}<500 D$, GLOBAL takes over solving the highest proportion of the problems. But for budgets larger than 500D, BIPOP-CMA-ES is the best algorithm, solving almost $100 \%$ of the problems, while the other algorithms solved about $65 \%$ of the problems with GLOBAL being fastest, followed by NEWUOA, MCS and DIRECT.

In the 20-D space, the differences start being more pronounced. For low evaluation budgets (\#FEs $<100 D$ ), NEWUOA holds the lead closely followed by MCS. For $100 D<\# \mathrm{FEs}<1000 D$, GLOBAL followed by NEWUOA are most successful. And again, for budgets larger than $1000 D$, BIPOP-CMA-ES is the best, solving about $92 \%$ of the problems, followed by NEWUOA, GLOBAL, MCS and DIRECT solving about 60, 50,40 , and $20 \%$ of the problems, respectively.

\subsection{Separable functions $f_{1}-f_{5}$}

The results for the separable functions $f_{1}-f_{5}$ are aggregated in the ECDF graphs of ERT for the 5-D and 20-D functions in Figs. 1 and 2, respectively, in the upper right part. The detailed results are presented in Table 1 for the 5-D functions, and in Table 2 for the 20-D functions.

In the 5-D space, for \#FEs $<10 D$, NEWUOA and MCS are very fast solving about $40 \%$ of the problems (these are the problems associated with $f_{1}$, sphere func- 
all functions

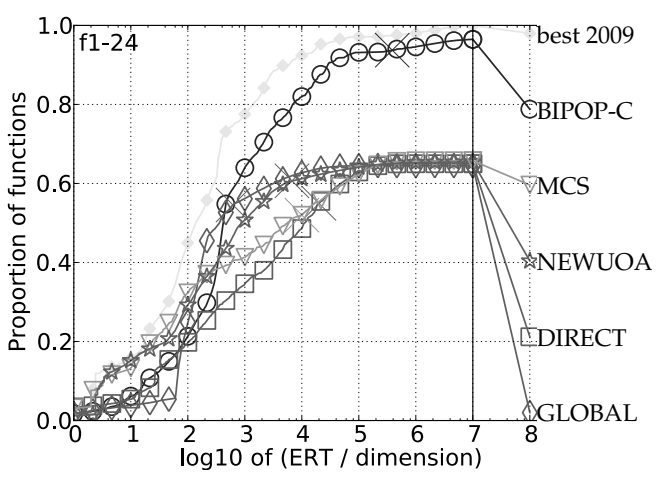

moderate fcts

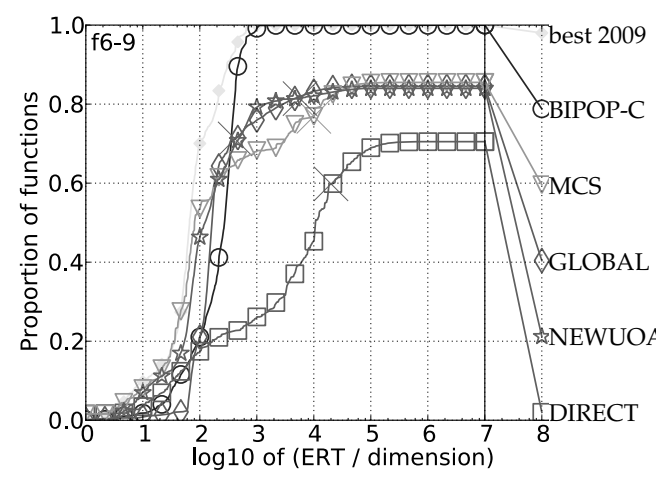
multi-modal fcts

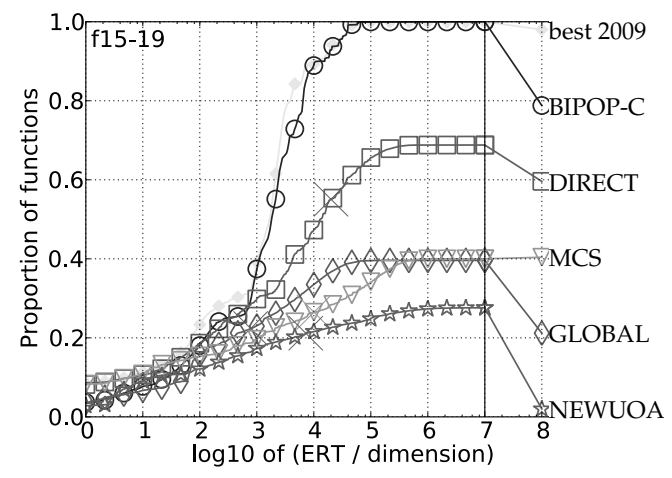

separable fcts

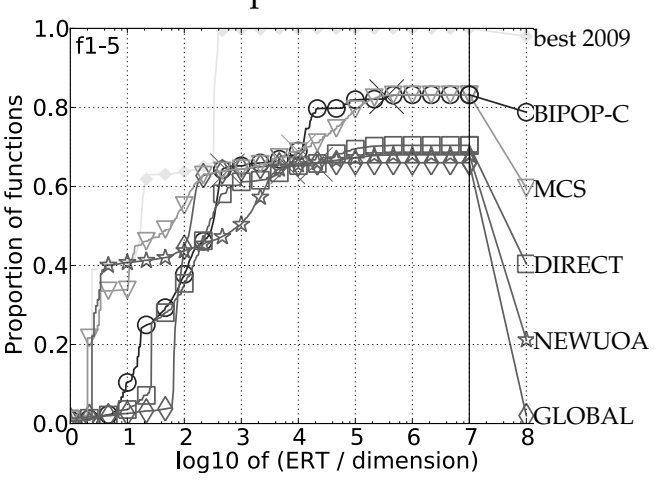

ill-conditioned fcts

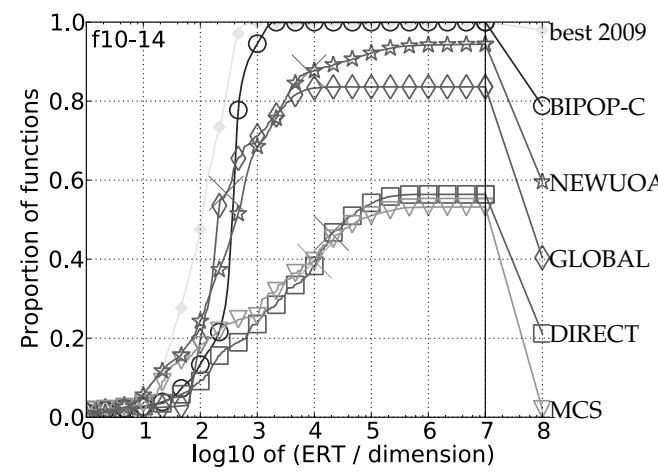

weak structure fcts

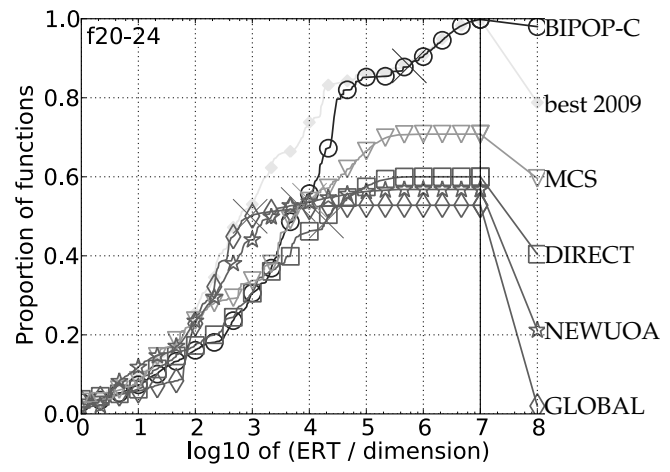

Figure 1: Empirical cumulative distribution of the bootstrapped distribution of ERT over dimension for 50 targets in $10^{[-8 . .2]}$ for all functions and subgroups in 5-D. The best ever line corresponds to the algorithms from BBOB-2009 with the best ERT for each of the targets considered. 
all functions

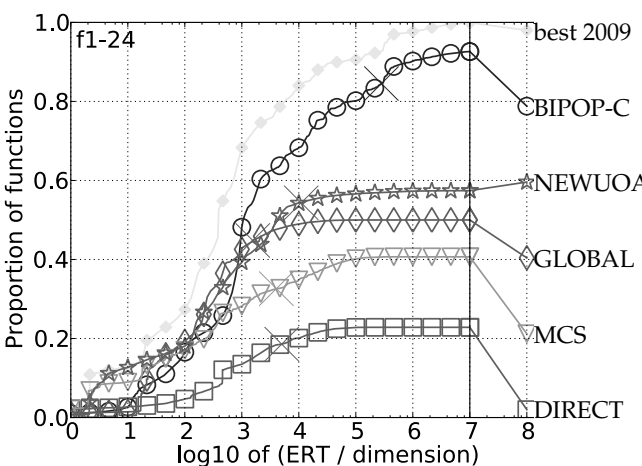

moderate fcts

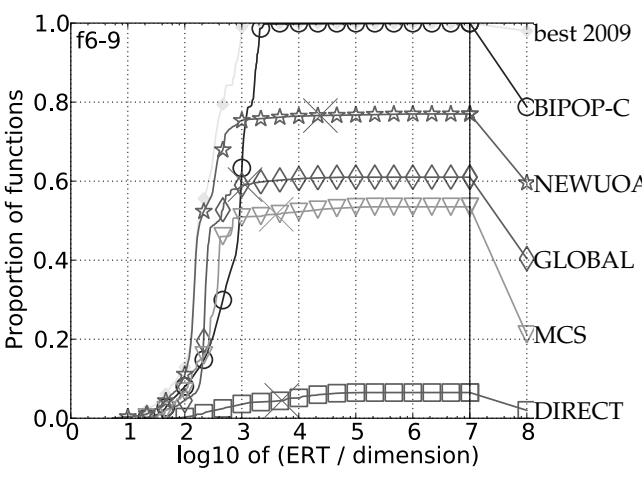
multi-modal fcts

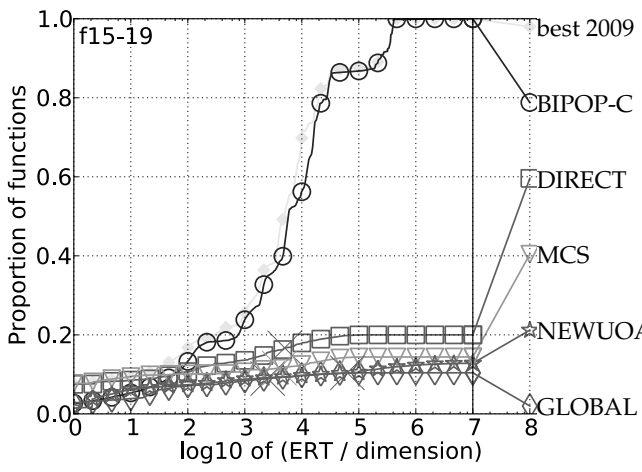

separable fcts

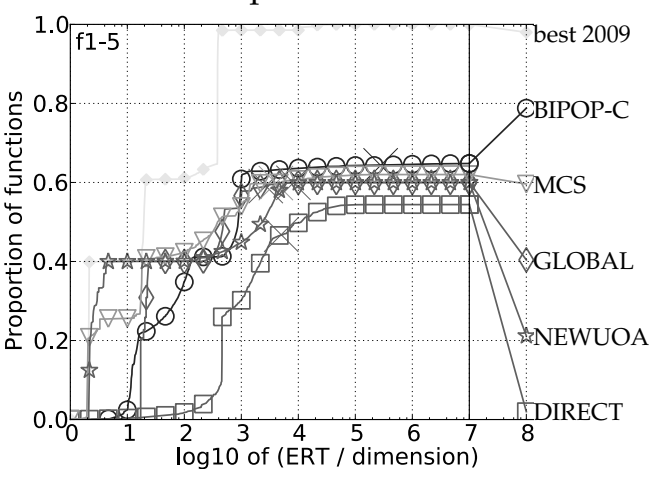

ill-conditioned fcts

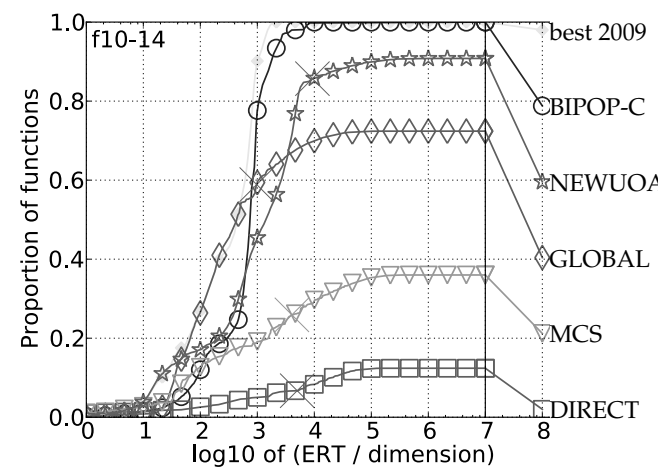

weak structure fcts

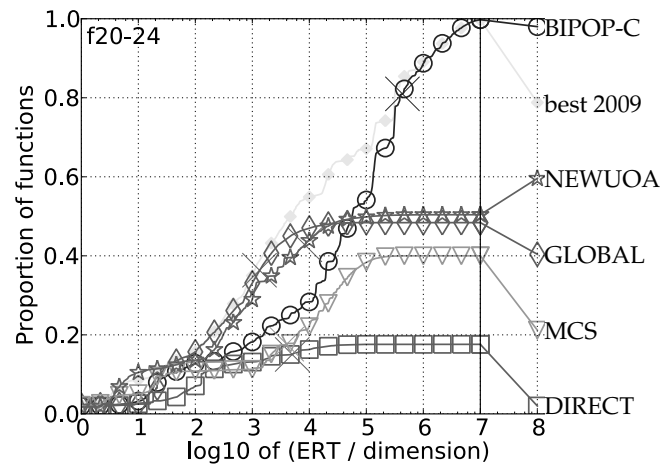

Figure 2: Empirical cumulative distribution of the bootstrapped distribution of ERT over dimension for 50 targets in $10^{[-8 . .2]}$ for all functions and subgroups in 20-D. The best ever line corresponds to the algorithms from BBOB-2009 with the best ERT for each of the targets considered. 
Table 1: ERT on $f_{1}-f_{5}$ in 5-D over $\mathrm{ERT}_{\text {best }}$ obtained in BBOB-2009

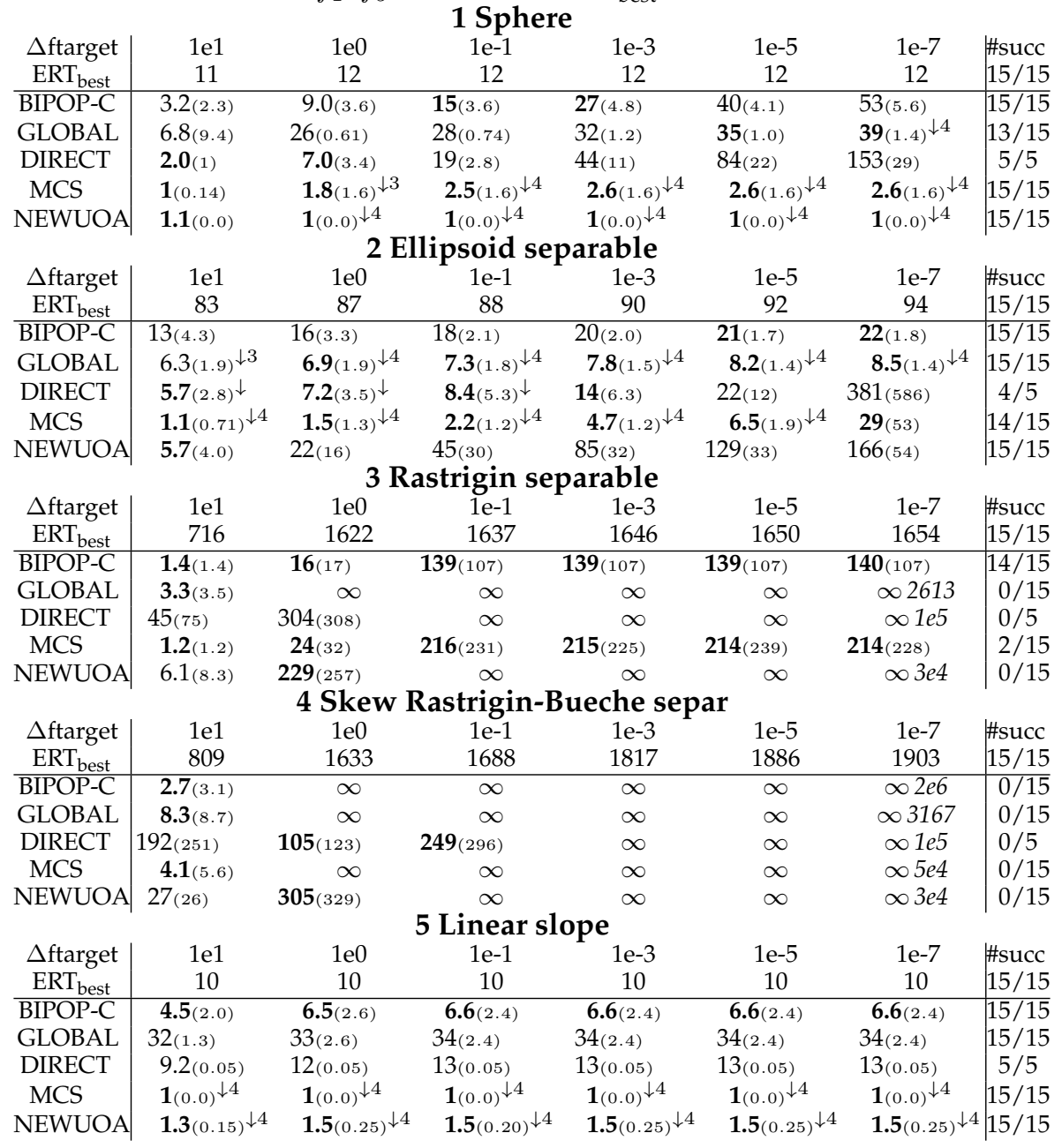

tion, and $f_{5}$, linear function), while the other algorithms solved only a few percent. Between $10 D-100 D$ evaluations, MCS beats NEWUOA reaching $60 \%$, and around $100 D$ evaluations, the other methods join the mainstream, solving about $40 \%$. For $100 D<\#$ FEs $<1000 D$, MCS, BIPOP-CMA-ES, GLOBAL, and DIRECT showed similar performance by solving about $60 \%$ of the problems, while NEWUOA seems to be the least successful here. For budgets larger than $10^{4} D$ evaluations, BIPOP-CMA-ES and MCS increase the percentage of the solved problems above $80 \%$ (with BIPOPCMA-ES being slightly faster). This increase seems to be caused by solving $f_{3}$, separable Rastrigin function, where GLOBAL, DIRECT and NEWUOA were able to solve only the problems with loose target levels, while BIPOP-CMA-ES and MCS were able to solve even the high precision problems. DIRECT, NEWUOA and GLOBAL eventually reached the level of $65 \%$ of the solved problems. About $15 \%$ of all the separable problems remained unsolved by any of the compared algorithms. To a large extent, these were the problems associated with $f_{4}$, separable multimodal Rastrigin-Büche function.

For the 20-D functions and a low evaluation budget (\#FEs $<10 D$ ), NEWUOA fol- 
Table 2: ERT on $f_{1}-f_{5}$ in 20-D over $\mathrm{ERT}_{\text {best }}$ obtained in BBOB-2009

\begin{tabular}{|c|c|c|c|c|c|c|c|}
\hline \multicolumn{8}{|c|}{1 Sphere } \\
\hline$\Delta$ ftarget & $1 \mathrm{e} 1$ & $1 \mathrm{e} 0$ & $1 e-1$ & $1 e-3$ & $1 e-5$ & $1 \mathrm{e}-7$ & \#succ \\
\hline $\mathrm{ERT}_{\text {best }}$ & 43 & 43 & 43 & 43 & 43 & 43 & $15 / 15$ \\
\hline$\overline{\mathrm{BIPOP}-\mathrm{C}}$ & $7.9_{(1.7)}$ & $14(2.8)$ & $20(2.0)$ & $33(3.5)$ & $45(3.0)$ & $57(3.1)$ & $15 / 15$ \\
\hline GLOBAL & $8.0(0.0)$ & $8.0(0.0)^{\downarrow 4}$ & $8.0(0.0)^{\downarrow 4}$ & $8.0(0.0)^{\downarrow 4}$ & $8.0(0.0)^{\downarrow 4}$ & $8.0(0.0)^{\downarrow 4}$ & $15 / 15$ \\
\hline DIRECT & $48(11)$ & $112(15)$ & $225(40)$ & $485(38)$ & $874(64)$ & 1393(98) & $4 / 5$ \\
\hline MCS & $2.4_{(2.8)^{\downarrow 3}}$ & $\mathbf{6 . 4}_{(3.5)^{\downarrow 4}}$ & $6.8(3.5)^{\downarrow 4}$ & $7.0(3.4)^{\downarrow 4}$ & $7.0_{(3.4)^{\downarrow 4}}^{\downarrow 4}$ & $7.0(3.4)^{\downarrow 4}$ & $15 / 15$ \\
\hline NEWUOA & $\mathbf{1 . 0}(0.02)^{\downarrow 4}$ & $\mathbf{1 . 0}(0.01)^{\downarrow 4}$ & $\mathbf{1 . 0}(0.01)^{\downarrow 4}$ & $\mathbf{1 . 0}(0.01)^{\downarrow 4}$ & $\mathbf{1 . 0}(0.01)^{\downarrow 4}$ & $\mathbf{1 . 0}(0.01)^{\downarrow}$ & $415 / 15$ \\
\hline \multicolumn{8}{|c|}{2 Ellipsoid separable } \\
\hline$\Delta$ ftarget & 1e1 & $1 \mathrm{e} 0$ & $1 e-1$ & $1 e-3$ & $1 e-5$ & $1 e-7$ & \#succ \\
\hline ERT $_{\text {best }}$ & 385 & 386 & 387 & 390 & 391 & 393 & $15 / 15$ \\
\hline BIPOP-C & $35(6.6)$ & $40(4.4)$ & $44(3.6)$ & $47(2.3)$ & $48(2.3)$ & $50(2.2)$ & $15 / 15$ \\
\hline GLOBAL & $18(3.7)^{\downarrow 4}$ & $23(3.0)^{\downarrow 3}$ & 26(13) & $33(14)$ & $51(40)$ & $63(65)$ & $13 / 15$ \\
\hline DIRECT & $134_{(133)}$ & $471_{(522)}$ & $487(520)$ & $537(516)$ & $\infty$ & $\infty 1 e 5$ & $0 / 5$ \\
\hline MCS & $\mathbf{5 . 4}(2.5)^{\downarrow 4}$ & $\mathbf{1 4}(7.6)^{\downarrow 4}$ & $\mathbf{2 1}(16)^{\downarrow 3}$ & $43(27)$ & $45(26)$ & $\infty 8 e 4$ & $0 / 15$ \\
\hline NEWUOA & $18(7.9)^{\downarrow 3}$ & $42(21)$ & $71_{(36)}$ & $125(43)$ & $174(51)$ & $\mathbf{2 1 9}(67)$ & $15 / 15$ \\
\hline \multicolumn{8}{|c|}{3 Rastrigin separable } \\
\hline$\Delta$ ftarget & 1e1 & $1 \mathrm{e} 0$ & 1e-1 & $1 e-3$ & $1 e-5$ & $1 \mathrm{e}-$ & Hsucc \\
\hline ERT & 5066 & 7626 & 7635 & 7643 & 7646 & 7651 & $15 / 15$ \\
\hline BIPOP-C & $12(7.1)$ & $\infty$ & $\infty$ & $\infty$ & $\infty$ & $\infty 6 e 6$ & $0 / 15$ \\
\hline GLOBAL & $\infty$ & $\infty$ & $\infty$ & $\infty$ & $\infty$ & $\infty 5$ & $0 / 15$ \\
\hline DIRECT & $\infty$ & $\infty$ & $\infty$ & $\infty$ & $\infty$ & $\infty 1$ & $0 / 5$ \\
\hline MCS & $28(32)$ & $\infty$ & $\infty$ & $\infty$ & $\infty$ & $\infty 8$ & $0 / 15$ \\
\hline NEWUOA & $\infty$ & $\infty$ & $\infty$ & $\infty$ & $\infty$ & $\infty 1 e 5$ & $0 / 15$ \\
\hline \multicolumn{8}{|c|}{4 Skew Rastrigin-Bueche separ } \\
\hline$\Delta \mathrm{fta}$ & 1e1 & $1 \mathrm{e} 0$ & $1 \mathrm{e}-1$ & $1 e-3$ & $1 \mathrm{e}^{-}$ & $1 e$ & \#succ \\
\hline ERT & 4722 & 7628 & 7666 & 7700 & 7758 & $1.41 \mathrm{e}$ & $9 / 15$ \\
\hline BIPOP-C & $\infty$ & $\infty$ & $\infty$ & $\infty$ & $\infty$ & $\infty 6$ & $0 / 15$ \\
\hline GLOH & $\infty$ & $\infty$ & $\infty$ & $\infty$ & $\alpha$ & $\infty 8$ & $0 / 15$ \\
\hline DIRE & $\infty$ & $\infty$ & $\infty$ & $\infty$ & $\alpha$ & $\infty 1$ & $0 / 5$ \\
\hline $\mathrm{MC}$ & $\infty$ & $\propto$ & $\propto$ & $\infty$ & $\alpha$ & $\infty \delta$ & $0 / 15$ \\
\hline NEWUOA & $\infty$ & $\infty$ & $\infty$ & $\infty$ & $\infty$ & $\infty 2 e 5$ & $0 / 15$ \\
\hline \multicolumn{8}{|c|}{5 Linear slope } \\
\hline$\Delta \mathrm{fta}$ & $1 \mathrm{e} 1$ & $1 \mathrm{e} 0$ & $1 \mathrm{e}-1$ & $1 e-3$ & $1 e-5$ & 1e-7 & tsucc \\
\hline ERT $_{\text {best }}$ & 41 & 41 & 41 & 41 & 41 & 41 & $15 / 15$ \\
\hline BIPOP-C & $\mathbf{1}(0.80)$ & $2(1.2)$ & $\mathbf{6 . 3}(1.2)$ & $\mathbf{6 . 3}(1.2)$ & $\mathbf{6 . 3}(1.2)$ & $\mathbf{6 . 3}(1.2)$ & $15 / 15$ \\
\hline GLOBAL & $10(0.52)$ & 8) & $11(0.78)$ & $11_{(0.78)}$ & $11(0.78)$ & $11(0.78)$ & $15 / 15$ \\
\hline DIRECT & & & & & $226(0.01)$ & $226(0.01)$ & $5 / 5$ \\
\hline MCS & $\mathbf{1}_{(0.01)^{\downarrow 4}}$ & $\mathbf{1}_{(0.01)} \downarrow 4$ & $\mathbf{1}_{(0.01)^{\downarrow 4}}^{\downarrow}$ & $\mathbf{1}_{(0.01)^{\downarrow 4}}$ & $\mathbf{1}_{(0.01)^{\downarrow 4}}$ & $\mathbf{1}_{(0.01)} \downarrow 4$ & $15 / 15$ \\
\hline NEWUOA & $\mathbf{1 . 2}(0.10)^{\downarrow 4}$ & $\mathbf{1 . 5}(0.38)^{\downarrow}$ & $\mathbf{1 . 6}(0.48)^{\downarrow 4}$ & $\mathbf{1 . 6}(0.48)^{\downarrow 4}$ & $\mathbf{1 . 6}(0.48)^{\downarrow 4}$ & $\mathbf{1 . 6}(0.48)^{\downarrow}$ & $415 / 15$ \\
\hline
\end{tabular}

lowed by MCS are the best. Between $20 D-100 D$, NEWUOA, MCS, and GLOBAL are equally good solving $40 \%$ of the problems (again, these are the problems associated with $f_{1}$ and $f_{5}$ ). For $100 D<\#$ FEs $<1000 D$, MCS, and GLOBAL hold their superiority, but BIPOP-CMA-ES joins them, while NEWUOA slows down. For \#FEs > 1000D, BIPOP-CMA-ES wins, solving eventually about $65 \%$ of the problems, followed by MCS, GLOBAL, and NEWUOA $(62,60$ and 60$) \%$. About $35 \%$ of the problems remain unsolved, these are associated mainly with the multimodal functions $f_{3}$ and $f_{4}$, which present considerable problems to any algorithm that does not assume separability.

\subsection{Unimodal functions with moderate conditioning $f_{6}-f_{9}$}

The results for the unimodal functions with moderate conditioning $f_{6}-f_{9}$ are aggregated in the ECDF graphs of ERT for the 5-D and 20-D functions in Figs. 1 and 2, respectively, in the middle left part. The detailed results are presented in Table 3 for the 5-D functions, and in Table 4 for the 20-D functions.

For the 5-D problems, MCS and NEWUOA are the most successful algorithms until 
P. Pošík, W. Huyer, and L. Pál

Table 3: ERT on $f_{6}-f_{9}$ in 5-D over ERT $_{\text {best }}$ obtained in BBOB-2009

\begin{tabular}{|c|c|c|c|c|c|c|c|}
\hline \multicolumn{8}{|c|}{6 Attractive sector } \\
\hline$\Delta$ ftarget & $1 \mathrm{e} 1$ & $1 \mathrm{e} 0$ & $1 e-1$ & $1 e-3$ & $1 e-5$ & $1 \mathrm{e}-7$ & Hsuc \\
\hline $\mathrm{ERT}_{\text {best }}$ & 114 & 214 & 281 & 580 & 1038 & 1332 & $15 /$ \\
\hline BIPOP-C & $\mathbf{2 . 3}(1.3)$ & 2.1 $(0.65)$ & $2.2(0.64)$ & $1.7_{(0.22)}$ & $1.3(0.28)$ & $\mathbf{1 . 3}(0.19)$ & $15 / 1$ \\
\hline GLOBAL & $\mathbf{2 . 9}(0.21)$ & 2.1 $(0.60)$ & $2.0(0.50)$ & $2.2(1.6)$ & $\mathbf{3 . 6}(3.6)$ & $35(37)$ & \\
\hline DIRECT & $2.3(1.6)$ & $28(21)$ & $789(712)$ & $\infty$ & $\infty$ & $\infty 1 e 5$ & 0 \\
\hline MCS & $2.7(3.9)$ & $47_{(117)}$ & 41 (93) & $71(86)$ & $46(56)$ & $54(64)$ & \\
\hline NEWUOA & $1.7_{(1.5)}$ & 2.4(1.3) & $3.6(2.2)$ & $3.3(1.7)$ & $2.7(1.2)$ & $2.9(1.1)$ & $|15|$ \\
\hline \multicolumn{8}{|c|}{7 Step-ellipsoid } \\
\hline$\Delta \mathrm{fta}$ & $1 \mathrm{e} 1$ & $1 \mathrm{e} 0$ & $1 \mathrm{e}-1$ & $1 e-3$ & $1 e-5$ & $1 \mathrm{e}-7$ & \\
\hline $\mathrm{ERT}_{\text {best }}$ & 24 & 324 & 1171 & 1572 & 1572 & 1597 & 15 \\
\hline BIPOP-C & $5.0(5.0)$ & $1.5(1.4)$ & $\mathbf{1}(1.2)$ & $\mathbf{1}(0.88)$ & $\mathbf{1}(0.88)$ & $\overline{1}(0.86)$ & 15 \\
\hline GLOBAL & $12(6.7)$ & $5.7(5.4)$ & $10(12)$ & $\infty$ & $\infty$ & $\infty 1880$ & \\
\hline DIRECT & $2.8(1.6)$ & $\mathbf{1 . 7}(1.1)$ & $115(146)$ & $\infty$ & $\infty$ & $\infty 1 e 5$ & \\
\hline MCS & $2.8(4.5)$ & $5.9(4.3)$ & $13(21)$ & $\infty$ & $\infty$ & $\infty 5 e 4$ & \\
\hline NEWUOA & $10(15)$ & $13(18)$ & $60_{(57)}$ & $\infty$ & $\infty$ & $\infty 3 e 4$ & $0 /$ \\
\hline \multicolumn{8}{|c|}{8 Rosenbrock original } \\
\hline$\Delta \mathrm{fta}$ & $1 \mathrm{e} 1$ & $1 \mathrm{e} 0$ & $1 \mathrm{e}-1$ & $1 e-3$ & $1 e-5$ & $1 e-7$ & \\
\hline ERT & 73 & 273 & 336 & 391 & 410 & 422 & 15 \\
\hline BIPOP-C & $3.2(1.3)$ & $3.7(2.3)$ & $4.5(1.9)$ & $4.8(1.7)$ & $5.1(1.7)$ & $5.4(1.6)$ & $15 /$ \\
\hline GLOBAL & $5.0(0.34)$ & $2.1(1.3)^{\downarrow}$ & 2.1(1.1) $\downarrow 2$ & $\mathbf{2 . 1}(0.86)^{\downarrow 3}$ & $2.1(0.86)^{\downarrow 3}$ & $2.2(0.81)^{\downarrow 4}$ & $15 /$ \\
\hline DIRECT & $4.1_{(2.9)}$ & $5.7(4.8)$ & $22(15)$ & $100(94)$ & 195 (185) & $293(288)$ & $3 / 5$ \\
\hline MCS & $\mathbf{1 . 5}(0.91)^{\downarrow 2}$ & $\mathbf{1 . 0}(1.2)^{\downarrow 3}$ & $\mathbf{1 . 0}(0.99)^{\downarrow 4}$ & $\mathbf{1 . 1}_{(0.89)^{\downarrow 4}}^{\downarrow 4}$ & $\mathbf{1 . 1}_{(0.86)^{\downarrow 4}}$ & $\mathbf{1 . 1}(0.84)^{\downarrow 4}$ & $15 / 1$ \\
\hline NEWUOA & $\mathbf{1}_{(0.95)^{\downarrow 3}}$ & $\mathbf{1 . 1}(0.81)^{\downarrow 2}$ & $1.2(0.49)^{\downarrow 3}$ & $1.2(0.44)^{\downarrow 4}$ & $1.2(0.41)^{\downarrow 4}$ & $1.2(0.40)^{\downarrow}$ & $15 /$ \\
\hline \multicolumn{8}{|c|}{9 Rosenbrock rotated } \\
\hline$\Delta$ ftarget & $1 \mathrm{e} 1$ & $1 \mathrm{e} 0$ & $1 e-1$ & $1 e-3$ & $1 e-5$ & $1 \mathrm{e}-7$ & \#suc \\
\hline $\mathrm{ERT}_{\text {best }}$ & 35 & 127 & 214 & 300 & 335 & 369 & $15 /$ \\
\hline BIPOP-C & $5.8(1.8)$ & $8.7(3.4)$ & $7.2(2.3)$ & $6.4(1.5)$ & $6.3(1.3)$ & $6.2(1.2)$ & $15 /$ \\
\hline GLOBAL & $11(2.2)$ & $4.6(1.3)$ & $3.2(0.80)^{\downarrow 2}$ & $2.8(0.74)^{\downarrow 2}$ & $2.7(0.60)^{\downarrow 3}$ & $2.7_{(1.2)} \downarrow 3$ & $13 /$ \\
\hline DIRECT & $3.2(2.2)$ & $4.2(2.3)$ & $48(38)$ & $134(87)$ & $151(79)$ & $306(300)$ & $3 / 5$ \\
\hline MCS & $\mathbf{1}_{(0.55)^{\downarrow 4}}^{\downarrow}$ & $\mathbf{1}_{(0.23)^{\downarrow 4}}$ & $\mathbf{1}_{(0.16)^{\downarrow 4}}$ & $\mathbf{1}_{(0.16)^{\downarrow 4}}$ & $\mathbf{1}_{(0.19)^{\downarrow 4}}$ & $\mathbf{1}_{(0.12)^{\downarrow 4}}^{\downarrow 4}$ & $15 / 1$ \\
\hline NEWUOA & $1.8(0.74)^{\downarrow 4}$ & $3.6(2.8)$ & $\mathbf{2 . 5}(1.6)^{\downarrow 2}$ & $1.9_{(1.1)}{ }^{\downarrow 3}$ & $\mathbf{1 . 9} 9_{(1.0)} \downarrow 3$ & $\mathbf{1 . 7}(0.92)^{\downarrow 4}$ & $15 / 1$ \\
\hline
\end{tabular}

$100 D$ evaluations. They gained their superiority by efficiently solving $f_{8}$ and $f_{9}$, the original and rotated Rosenbrock functions. For \#FEs $<50 D$, DIRECT is third behind them, while GLOBAL solves only a very low proportion of the problems (due to the initial random phase). Around $100 \mathrm{D}$ evaluations, GLOBAL makes a huge progress (by executing the local search, Nelder-Mead in case of 5-D) and joins the leading group. For a short range of evaluation budgets, it becomes the best competitor. DIRECT slows down solving only about $20 \%$ of the problems where the others already solved around $60 \%$. For \#FEs > 500D, BIPOP-CMA-ES clearly wins (solving eventually all problems, for which it needed about $1000 D$ evaluations). MCS, GLOBAL, and NEWUOA solved about $85 \%$ of the problems. The problems they did not solve are associated with $f_{7}$, step-ellipsoid function, where they were not able to find the target levels $10^{-3}$ and tighter. DIRECT was the slowest and least successful solving only $70 \%$ of the problems. Additionally to $f_{7}$, it also did not solve the $f_{6}$, attractive sector function.

In the 20-D space, for low evaluation budgets (\#FEs $<100 D$ ), NEWUOA starts successfully by solving about $10 \%$ of the problems, followed by BIPOP-CMA-ES, MCS, and GLOBAL (all between 5 and 10\%). DIRECT solved virtually no problem so far. For $100 D<\#$ FEs $<1000 D$, NEWUOA stays in the lead, solving more than $75 \%$ of the problems, with GLOBAL and MCS behind (60\% and over $50 \%$, respectively). For larger budgets (\#FEs > 1000D), NEWUOA, GLOBAL, and MCS did not improve their results significantly. Around $1000 D$ evaluations, the proportion of the problems solved by BIPOP-CMA-ES rises steeply and reaches $100 \%$ after $2000 D$ evaluations. DIRECT 
Table 4: ERT on $f_{6}-f_{9}$ in 20-D over ERT best $_{6}$ obtained in BBOB-2009

\begin{tabular}{|c|c|c|c|c|c|c|c|}
\hline \multicolumn{8}{|c|}{6 Attractive sector } \\
\hline$\Delta$ ftarget & $1 \mathrm{e} 1$ & $1 \mathrm{e} 0$ & $1 e-1$ & $1 e-3$ & $1 e-5$ & $1 \mathrm{e}-7$ & \#succ \\
\hline ERT $_{\text {best }}$ & 1296 & 2343 & 3413 & 5220 & 6728 & 8409 & $15 / 15$ \\
\hline BIPOP-C & $\mathbf{1 . 5}(0.38)$ & $.3(0.25)$ & $1.2(0.23)$ & $\mathbf{1 . 1}(0.18)$ & $\mathbf{1 . 2}(0.12)$ & $\mathbf{1 . 2}(0.10)$ & $15 / 15$ \\
\hline GLOBAL & $3.6(1.00)$ & $3.6(0.74)$ & 6.1(3.0) & $\infty$ & $\infty$ & $\infty 4 e 4$ & $0 / 15$ \\
\hline DIRECT & $\infty$ & $\infty$ & $\infty$ & $\infty$ & $\infty$ & $\infty 1 e 5$ & $0 / 5$ \\
\hline MCS & $\infty$ & $\infty$ & $\infty$ & $\infty$ & $\infty$ & $\infty 8 e 4$ & $0 / 15$ \\
\hline NEWUOA & $\mathbf{1}(0.33)$ & $\mathbf{1}_{(0.37)}$ & $\mathbf{1}_{(0.49)}$ & 1.1 $(0$ & $\mathbf{1 . 3}(0.83)$ & $\mathbf{1 . 3}(0.66)$ & $15 / 15$ \\
\hline \multicolumn{8}{|c|}{7 Step-ellipsoid } \\
\hline$\Delta \mathrm{ftc}_{\mathrm{c}}$ & $1 \mathrm{e} 1$ & $1 \mathrm{e} 0$ & $1 e-1$ & $1 e-3$ & $1 \mathrm{e}$ & $1 \mathrm{e}-7$ & \\
\hline $\mathrm{ERT}_{\text {best }}$ & 1351 & 4274 & 9503 & 16524 & 16524 & 16969 & $15 / 15$ \\
\hline BIPOP-C & $\mathbf{1}_{(0.47)}$ & $4.9(2.5)$ & $3.5(0.60)$ & $2.2(0.26)$ & $2.2(0.26)$ & $2.1(0.25)$ & $15 / 15$ \\
\hline GLO & $\infty$ & $\infty$ & $\infty$ & $\infty$ & $\infty$ & $\infty 1 e 4$ & $0 / 15$ \\
\hline DIR & $\infty$ & $\infty$ & $\infty$ & $\infty$ & $\alpha$ & $\infty 1 e 5$ & $0 / 5$ \\
\hline $\mathrm{MC}$ & $\infty$ & $\infty$ & $\infty$ & $\infty$ & $\infty$ & $\infty 8 e 4$ & $0 / 15$ \\
\hline NEW & $\infty$ & $\infty$ & $\infty$ & $\infty$ & $\infty$ & $\infty 5 e 5$ & $0 / 15$ \\
\hline \multicolumn{8}{|c|}{8 Rosenbrock original } \\
\hline$\Delta \mathrm{ft}$ & & $1 \mathrm{e} 0$ & $1 e-1$ & $1 \mathrm{e}-3$ & $1 \mathrm{e}$ & $1 \mathrm{e}-7$ & \\
\hline ER & 2039 & 3871 & 4040 & 4219 & 4371 & 4484 & 15 \\
\hline BIPOP-C & $4.0_{(1.0)}$ & $4.0_{(0.68)}$ & $4.3(0.62)$ & $4.5(0.60)$ & $4.6(0.56)$ & $4.6(0.56)$ & $15 / 15$ \\
\hline GLO & $1.6(0.32)^{\downarrow}$ & $\mathbf{1 . 2}(0.16)^{\downarrow}$ & $1.2(0.16) \downarrow 4$ & $1.2(0.15)^{\downarrow}$ & $1.2(0.14)^{\downarrow}$ & $1.2_{(0.14)^{\downarrow}}$ & $15 / 15$ \\
\hline DIRECT & $\infty$ & $\infty$ & $\infty$ & $\infty$ & $\infty$ & $\infty 1 e 5$ & $0 / 5$ \\
\hline MCS & $\mathbf{1 . 5}(0.78)^{\downarrow 4}$ & $\mathbf{1 . 7} 7_{(1.4)^{\downarrow 3}}$ & $\mathbf{1 . 8}(1.4)^{\downarrow 3}$ & $\mathbf{1 . 8}(1.4)^{\downarrow 3}$ & $\mathbf{1 . 8}(1.3)^{\downarrow 3}$ & $1.8_{(1.3)} \downarrow 3$ & $15 / 15$ \\
\hline NEWUOA & $\mathbf{1}(0.26)^{\downarrow 4}$ & $\mathbf{1}_{(0.59)^{\downarrow 4}}$ & $\mathbf{1}(0.56)^{\downarrow 4}$ & $\mathbf{1}_{(0.54)^{\downarrow}}^{\downarrow 4}$ & $\mathbf{1}_{(0.52)} \downarrow 4$ & $\mathbf{1}_{(0.50)} \downarrow 4$ & $15 / 15$ \\
\hline \multicolumn{8}{|c|}{9 Rosenbrock rotated } \\
\hline$\Delta \mathrm{fta}$ & 1 & $1 \mathrm{e} 0$ & $1 e-1$ & $1 e-3$ & $1 e-5$ & $1 \mathrm{e}-7$ & \#succ \\
\hline $\mathrm{ERT}_{\text {best }}$ & 1716 & 3102 & 3277 & 3455 & 3594 & 3727 & $15 / 15$ \\
\hline BIPOP-C & $4.7(1.7)$ & $5.7_{(1.1)}$ & $6.0_{(1.1)}$ & $6.1(1.0)$ & $6.1_{(1.00)}$ & $6.1(0.93)$ & $15 / 15$ \\
\hline GLOBAL & $1.7(0.28)^{\downarrow}$ & $\mathbf{1 . 7}(0.89)$ & $\mathbf{1 . 6}(0.84)^{\downarrow}$ & $\mathbf{1 . 6}(0.79)^{\downarrow}$ & $1.6(0.77)$ & $\mathbf{1 . 5}(0.74)^{\downarrow 4}$ & $15 / 15$ \\
\hline DIRECT & $\infty$ & $\infty$ & $\infty$ & $\infty$ & $\infty$ & $\infty 1 e 5$ & $0 / 5$ \\
\hline MCS & $\mathbf{1}_{(0.48)^{\downarrow 4}}^{\downarrow}$ & $\mathbf{1 . 3}(0.68)^{\downarrow 4}$ & $1.5(0.70)^{\downarrow 4}$ & $\mathbf{1 . 6}(0.73)^{\downarrow 4}$ & $1.7(0.71)^{\downarrow 4}$ & $\mathbf{1 . 6}(0.68)^{\downarrow 4}$ & $15 / 15$ \\
\hline NEWUOA & $1.0(0.16)^{\downarrow}$ & $\mathbf{1}_{(0.60)^{\downarrow 4}}$ & $\mathbf{1}_{(0.57)^{\downarrow 4}}^{\downarrow 4}$ & $\mathbf{1}_{(0.54)^{\downarrow 4}}^{\downarrow 4}$ & $\mathbf{1}_{(0.52)^{\downarrow 4}}^{\downarrow 4}$ & $\mathbf{1}_{(0.50)^{\downarrow 4}}^{\downarrow 4}$ & $15 / 1$ \\
\hline
\end{tabular}

solved less than $10 \%$ of the problems. All algorithms (with the exception of DIRECT) are pretty efficient at solving the Rosenbrock functions, $f_{8}$ and $f_{9}$, thanks to the local search routines they use. The step-ellipsoid function, $f_{7}$, is even harder in 20-D, since only BIPOP-CMA-ES was able to solve the function to all reported target levels. The attractive sector function, $f_{6}$, was successfully solved by BIPOP-CMA-ES and NEWUOA only, GLOBAL was able to find target levels until $10^{-1}$, while DIRECT and MCS did not find any reported target level.

\subsection{Unimodal ill-conditioned functions $f_{10}-f_{14}$}

The results for the unimodal ill-conditioned functions $f_{10}-f_{14}$ are aggregated in the ECDF graphs of ERT for the 5-D and 20-D functions in Figs. 1 and 2, respectively, in the middle right part. The detailed results are presented in Table 5 for the 5-D functions, and in Table 6 for the 20-D functions.

Compared to the results of functions with moderate conditioning, on the illconditioned functions in the 5-D space the situation is quite similar with the exceptions that MCS and DIRECT have worse performance, while NEWUOA improved its results. For budgets lower than $100 D$ evaluations, NEWUOA and MCS are in the lead having solved about $20 \%$ of the problems. Around $100 \mathrm{D}$ evaluations, the performance of MCS starts to decline, while the performance of GLOBAL and BIPOP-CMA-ES rises steeply. For a short range of evaluation budgets, GLOBAL is the best competitor. It reaches the level of $50 \%$ twice as fast as NEWUOA or BIPOP-CMA-ES. For \#FEs > 500D, BIPOP- 
Table 5: ERT on $f_{10}-f_{14}$ in 5-D over ERT $\mathrm{E}_{\text {best }}$ obtained in BBOB-2009

\begin{tabular}{|c|c|c|c|c|c|c|c|}
\hline \multicolumn{8}{|c|}{ Ellipsoid } \\
\hline$\Delta$ ftarget & 1e1 & $1 \mathrm{e} 0$ & $1 e-1$ & $1 e-3$ & 1e-5 & $1 \mathrm{e}-7$ & \#suc \\
\hline ERT $_{\text {best }}$ & 349 & 500 & 574 & 626 & 829 & 880 & $15 / 15$ \\
\hline BIPOP-C & $3.5(0.82)$ & $2 . \mathbf{9}_{(0.43)}$ & $2.7(0.37)$ & $\mathbf{2 . 8}(0.25)$ & $2.3(0.18)$ & $2.4(0.14)$ & $15 / 15$ \\
\hline GLOBAL & $1.9_{(0.70)} \downarrow 3$ & $\mathbf{1 . 6}(0.49)^{\downarrow 3}$ & $1.8(0.71)$ & $2.0(1.5)$ & $\mathbf{1 . 7} \mathbf{7}_{(1.1)}$ & $\mathbf{1 . 7}(1.1)$ & $15 / 15$ \\
\hline DIRECT & $110_{(158)}$ & $141_{(181)}$ & $281(357)$ & $\infty$ & $\infty$ & $\infty 1 e 5$ & $0 / 5$ \\
\hline MCS & $277(320)$ & $\infty$ & $\infty$ & $\infty$ & $\infty$ & $\infty 5 e 4$ & $0 /$ \\
\hline \multicolumn{8}{|c|}{11 Discus } \\
\hline$\Delta$ ftarget & $1 \mathrm{e} 1$ & $1 \mathrm{e} 0$ & 1e-1 & $1 e-3$ & $1 e-5$ & $1 e-7$ & \#su \\
\hline ERT $_{\text {best }}$ & 143 & 202 & 763 & 1177 & 1467 & 1673 & $15 / 1$ \\
\hline BIPOP-C & $8.4(3.0)$ & $7.2(1.6)$ & $2.2(0.30)$ & $\mathbf{1 . 6}(0.20)$ & $\mathbf{1 . 4}(0.11)$ & $\mathbf{1 . 3}(0.11)$ & $15 / 15$ \\
\hline GLOBAL & $4.0_{(1.5)^{\downarrow 3}}$ & $\mathbf{5 . 5}(2.6)$ & $\mathbf{3 . 5}(3.2)$ & $\mathbf{5 . 0}(6.1)$ & 5.0 & $8.5(7.9)$ & $8 / 15$ \\
\hline DIRECT & $87_{(162)}$ & $2228(247$ & $\infty$ & $\infty$ & $\infty$ & $\infty 1 e 5$ & $0 / 5$ \\
\hline MCS & $82(106)$ & 61(501) & $\infty$ & $\infty$ & $\infty$ & $\infty 5 e 4$ & $0 / 15$ \\
\hline NEWUOA & $3.5(1.8)^{\downarrow 3}$ & $4.7(2.2)^{\downarrow}$ & 12 Bent cigar & gar $^{1.8(0.44)}$ & 2.0 & $2.2(0.41)$ & $15 / 15$ \\
\hline$\Delta$ ftarget & 1e1 & $1 \mathrm{e} 0$ & $1 \mathrm{e}-1$ & $1 e-3$ & $1 \mathrm{e}$ & $1 e-7$ & \#su \\
\hline ERT $_{\text {best }}$ & 108 & 268 & 371 & 461 & 1303 & 1494 & $15 / 15$ \\
\hline$\overline{\text { BIPOP-C }}$ & $11_{(12)}$ & 7.4(7.8) & 7.4(5.7) & $7.7(5.4)$ & $3.3(2.2$ & $\mathbf{3 . 3}(2.1)$ & $15 / 1$ \\
\hline GLOBAL & 4.6 $(1$ & $2.7(0.61)^{\downarrow}$ & $2.4(0.82)^{\downarrow 3}$ & $\mathbf{5 . 0}(6.7)^{\downarrow}$ & $3.1(4.0)^{\downarrow}$ & $3.4(4.0)$ & $6 / 15$ \\
\hline DIRECT & $8.5(1$. & $8.7(5.7)$ & $19(17)$ & $108(62)$ & $377(384)$ & $\infty 1 e 5$ & $0 / 5$ \\
\hline MCS & $\mathbf{1}(0.20)^{\downarrow 4}$ & $18(47)$ & $17(33)$ & $22(32)$ & $26(38)$ & $56(69)$ & $6 / 15$ \\
\hline \multicolumn{8}{|c|}{13 Sharp ridge } \\
\hline$\Delta \mathrm{ft}$ & 1 & $1 \mathrm{e}$ & $1 e-1$ & $1 \mathrm{e}$ & & $1 \epsilon$ & \\
\hline ER & 132 & 195 & 250 & 131 & 175 & 2255 & $15 / 15$ \\
\hline $\mathrm{BIPO}$ & $3.9_{3}$ & 5.4 & $\mathbf{5 . 9}(2.6)$ & $1.6(0)$ & 1.5 & 1.7 & $15 / 15$ \\
\hline GLO & 4.2 & $1(4$ & 11 & $\infty$ & $\infty$ & $\infty 1262$ & $0 / 15$ \\
\hline DIRECT & $7.0_{(5.2}$ & $21_{(15)}$ & $34_{(15)}$ & $42(46)$ & 19 (115) & $\infty 1 e 5$ & $0 / 5$ \\
\hline A & $41_{(5}$ & $14(2$ & 462 & $550(611)$ & $\alpha$ & $5 e 4$ & $0 / 15$ \\
\hline NEWUOA & $3.1(3.3)$ & $9.3(12)$ & $35(36)$ & $54(54)$ & $335(367)$ & $\infty 4 e 4$ & $0 / 1$ \\
\hline \multicolumn{8}{|c|}{14 Sum of different powers } \\
\hline$\Delta \mathrm{ft}$ & $1 \mathrm{e}$ & $1 \mathrm{e} 0$ & 1e-1 & $1 e-3$ & $1 \mathrm{e}$ & $1 e-7$ & Hs \\
\hline ERT $_{\text {best }}$ & 10 & 41 & 58 & 139 & 251 & 476 & $15 / 15$ \\
\hline BIPOP-C & 1.1(0.97) & $\mathbf{2 . 8}(1.3)$ & 3.7(0.91) & $4.6(0.73)$ & $5.4(0.55)$ & $4.5(0.34)$ & $15 / 15$ \\
\hline GLOBAL & $\mathbf{2 . 2}(1.9)$ & $7.7_{(0 .}$ & $5.9(0.28)$ & $\mathbf{3 . 3}(0.40)^{\downarrow 2}$ & $\mathbf{3 . 6}(2.5)^{\downarrow 3}$ & $\infty 1302$ & $0 / 15$ \\
\hline DIRE & $\mathbf{1}_{(1.4)}$ & $3.7_{(3.1)}$ & $4.8(3.5)$ & $23(25)$ & $1892_{(1990)}$ & $\infty 1 e 5$ & $0 / 5$ \\
\hline MCS & $1.4(2.3)$ & $2.8(3.3)$ & $2.7_{(2.3)}$ & $2.8(1.1)^{\downarrow 3}$ & $225(299)$ & $\infty 5 e 4$ & $0 / 15$ \\
\hline NEWUOA & $1.7(0.56)$ & $\mathbf{1}_{(0.35)^{\downarrow 4}}^{\downarrow 4}$ & $\mathbf{1}_{(0.27)^{\downarrow 4}}^{\downarrow 4}$ & $\mathbf{1 . 2}(0.31)^{\downarrow 4}$ & $\mathbf{5 . 5}(1.9)$ & 2525 & $0 / 1$ \\
\hline
\end{tabular}

CMA-ES takes over and solves all the problems needing slightly above $1000 \mathrm{D}$ evaluations (behavior very similar to functions with moderate conditioning). NEWUOA reaches almost $95 \%$ of the solved problems, GLOBAL $85 \%$, DIRECT and MCS around $55 \%$. DIRECT and MCS fail significantly on $f_{10}$, ellipsoid, and $f_{11}$, discus, functions. Note that on $f_{10}$, DIRECT can find the target level $10^{-1}$, while MCS finds only $10^{1}$, because the local search procedure employed by MCS cannot solve this function due to the problems with high-conditioning (see also Sec. 7.2). GLOBAL, on the other hand, has the biggest problems with $f_{13}$, sharp ridge, due to the inability of the GLOBAL's local search procedure (Nelder-Mead in case of 5-D) to make progress on this function. The tiny proportion of the problems not solved by NEWUOA consists of the problems with tight target levels $\left(<10^{-5}\right)$ associated mainly with $f_{13}$, sharp ridge.

In all previous cases, MCS belongs among 2 best algorithms with respect to the initial stage of the search. For the 20-D ill-conditioned functions, the initial performance of MCS is not that good. For \#FEs $<40 D$, the NEWUOA is the only algorithm having solved about $10 \%$ of the problems, while the others solved only a few percent so far. For 
A Comparison of Global Search Algorithms

Table 6: ERT on $f_{10}-f_{14}$ in 20-D over $\mathrm{ERT}_{\text {best }}$ obtained in BBOB-2009

\begin{tabular}{|c|c|c|c|c|c|c|c|}
\hline & & & $\mathrm{El}$ & & & & \\
\hline ERT & 7413 & 8661 & $10-1$ & (6) & & & \#SUCC \\
\hline & & & $10 / 35$ & $\frac{1}{2}$ & $170 / 3$ & 11746 & $15 / 15$ \\
\hline BIPOP-C & $\mathbf{1 . 9}(0.21)$ & $\mathbf{1 . 8}(0.17)$ & $\mathbf{1 . 6}(0.13)$ & $1.2(0.03)$ & 1.1(0.03) & $\mathbf{1 . 1}(0.03)$ & $15 / 15$ \\
\hline GLOBAL & $\mathbf{1}_{(0.22)^{\downarrow 4}}$ & $\mathbf{1 . 1}(0.15)$ & $\mathbf{1 . 1}(0.53)$ & $\mathbf{2 . 0}(1.7)$ & $\mathbf{5 . 9}(6.2)$ & $\infty 4 e 4$ & $0 / 15$ \\
\hline DIRECT & $\infty$ & $\infty$ & $\infty$ & $\infty$ & $\infty$ & $\infty 1 e 5$ & $0 / 5$ \\
\hline MCS & $\infty$ & $\infty$ & $\infty$ & $\infty$ & $\infty$ & $\infty 8 e 4$ & $0 / 15$ \\
\hline NEWUOA & $\mathbf{1 . 7}(0.50)$ & $2.6(0.78)$ & $11 \stackrel{3.3(1.1)}{\text { Discus }}$ & $\mathbf{4 . 0}(0.83)$ & $4.7(0.76)$ & $\mathbf{5 . 8}(1.0)$ & $15 / 15$ \\
\hline$\Delta$ ftarget & $1 \mathrm{e} 1$ & $1 \mathrm{e} 0$ & $1 e-1$ & $1 e-3$ & $1 e-5$ & $1 \mathrm{e}-7$ & \#succ \\
\hline ERT $_{\text {best }}$ & 1002 & 2228 & 6278 & 9762 & 12285 & 14831 & $15 / 15$ \\
\hline BIPOP-C & $\mathbf{1 0}(0.54)$ & $5.1(0.25)$ & 1.9(0.08) & $1.4(0.05)$ & $1.2(0.04)$ & $1.0(0.03)$ & $15 / 15$ \\
\hline GLOBAL & $1.2(0.42)^{\downarrow 4}$ & $\mathbf{1 . 0}(0.60)^{\downarrow 4}$ & $\mathbf{1}(0.84)$ & $\infty$ & $\infty$ & $\infty 3 e 4$ & $0 / 15$ \\
\hline DIRECT & $\infty$ & $\infty$ & $\infty$ & $\infty$ & $\infty$ & $\infty 1 e 5$ & $0 / 5$ \\
\hline MCs & $\infty$ & $\infty$ & $\infty$ & $\infty$ & $\infty$ & $\infty 8 e 4$ & $0 / 15$ \\
\hline NEWUOA & \multicolumn{6}{|c|}{12 Bent cigar } & $15 / 15$ \\
\hline$\Delta$ ftarget & $1 \mathrm{e} 1$ & $1 \mathrm{e} 0$ & $1 e-1$ & $1 e-3$ & $1 e-5$ & $1 \mathrm{e}-7$ & \#succ \\
\hline ERT $_{\text {best }}$ & 1042 & 1938 & 2740 & 4140 & 12407 & 13827 & $15 / 15$ \\
\hline BIPOP-C & $3.0_{(1.8)}$ & $4.0(3.2)$ & $4.5(3.3)$ & $4.5(2.1)$ & $\mathbf{1 . 9}(0.69)$ & $2.0(0.66)$ & $15 / 15$ \\
\hline GLOBAL & $\mathbf{1}(0.85)^{\downarrow 2}$ & $\mathbf{1}(0.88)^{\downarrow}$ & $\mathbf{1}_{(0.70)^{\downarrow 2}}$ & $\mathbf{1}_{(0.49)^{\downarrow 3}}$ & 1.1 $(1.1)^{\downarrow 3}$ & $3.4(3.2)$ & $0 / 15$ \\
\hline DIRECT & $421(481)$ & $235(258)$ & $\infty$ & $\infty$ & $\infty$ & $\infty 1 e 5$ & $0 / 5$ \\
\hline MCS & $\mathbf{1 . 1}(0.78)^{\downarrow}$ & $8.4(18)$ & $12(15)$ & $43(43)$ & $94_{(101)}$ & $\infty 8 e 4$ & $0 / 15$ \\
\hline NEWUOA & $3.0(2.9)$ & $3.0(2.4)$ & $3.0(1.7)$ & $\mathbf{2 . 5}(1.2)$ & $\mathbf{1}_{(0.42)^{\downarrow 2}}$ & $\mathbf{1}_{(0.37)^{\downarrow 3}}$ & 15/15 \\
\hline \multicolumn{8}{|c|}{13 Sharp ridge } \\
\hline$\Delta$ ftarget & $1 \mathrm{e} 1$ & $1 \mathrm{e} 0$ & $1 \mathrm{e}-1$ & $1 e-3$ & 1e-5 & $1 e-7$ & \#succ \\
\hline ERT $_{\text {best }}$ & 652 & 2021 & 2751 & 18749 & 24455 & 30201 & $15 / 15$ \\
\hline BIPOP-C & $4.3(5.6)$ & $2.7(2.1)$ & 5.1(5.6) & $1.5(0.77)$ & $2.3(2.3)$ & $3.0(1.9)$ & $15 / 15$ \\
\hline GLOBAL & $2.0(0.34)^{\downarrow 2}$ & 1.1(0.08) & $\mathbf{1 . 1}(0.04)$ & $4.5(5.1)$ & $\infty$ & $\infty 2 e 4$ & $0 / 15$ \\
\hline DIRECT & $\infty$ & $\infty$ & $\infty$ & $\infty$ & $\infty$ & $\infty 1 e 5$ & $0 / 5$ \\
\hline MCS & $34(46)$ & $37(30)$ & $61_{(60)}$ & $\infty$ & $\infty$ & $\infty 8 e 4$ & $0 / 15$ \\
\hline \multicolumn{7}{|c|}{14 Sum of different powers } & $0 / 15$ \\
\hline$\Delta$ ftarget & $1 \mathrm{e} 1$ & $1 \mathrm{e} 0$ & $1 \mathrm{e}-1$ & $1 e-3$ & $1 e-5$ & $1 e-7$ & \#succ \\
\hline ERT $_{\text {best }}$ & 75 & 239 & 304 & 932 & 1648 & 15661 & $15 / 15$ \\
\hline BIPOP-C & $3.9_{(1.2)}$ & $2.9(0.44)$ & $3.7(0.42)$ & $4.1(0.32)$ & $6.2(0.49)$ & $1.2(0.09)$ & $15 / 15$ \\
\hline GLOBAL & $5.0(0.28)$ & $2.2(0.22)^{\downarrow 3}$ & 2.1 $(0.21)^{\downarrow 4}$ & $\mathbf{1 . 1}(0.08)^{\downarrow} 4$ & $\mathbf{1}_{(0.04)^{\downarrow}}^{\downarrow 4}$ & $\infty 8538$ & $0 / 15$ \\
\hline DIRECT & $8.4(6.0)$ & $153(213)$ & $290(380)$ & $\infty$ & $\infty$ & $\infty 1 e 5$ & $0 / 5$ \\
\hline MCS & $\mathbf{1}(0.54)^{\downarrow 4}$ & $2.1(0.78)^{\downarrow}$ & 3.4 $(0.87)$ & 3.2 $(0.87)^{\downarrow}$ & $\infty$ & $\infty 8 e 4$ & $0 / 15$ \\
\hline NEWUOA & $\mathbf{1 . 5}(0.75)^{\downarrow 3}$ & $\mathbf{1}_{(0.32)^{\downarrow 4}}$ & $\mathbf{1}_{(0.28)^{\downarrow 4}}$ & $\mathbf{1}(0.18)^{\downarrow 4}$ & $\mathbf{9 . 1}(0.95)$ & $43(32)$ & $0 / 15$ \\
\hline
\end{tabular}

$40 D<\#$ FEs $<400 D$ range, GLOBAL is the most successful algorithm having solved about $60 \%$ of the problems, followed by NEWUOA, BIPOP-CMA-ES, and MCS (all between 20 and $30 \%$ ). For budgets above $1000 D$ evaluations, BIPOP-CMA-ES is again the best algorithm reaching $100 \%$ of the solved problems (again, it has behavior similar to behavior on moderate functions). NEWUOA solved more than $90 \%$ of the problems, beating GLOBAL by $20 \%$. MCS (37\%) and DIRECT (12\%) are not successful solvers for this class of the problems. NEWUOA has slight difficulties solving $f_{13}$, sharp ridge, to the target levels tighter than $10^{-3}$. On the other hand, among all BBOB competitors it is the fastest algorithm that solves $f_{12}$, bent cigar, for the tightest target levels below $10^{-3}$, and for the middle target levels between $10^{0}$ and $10^{-5}$ for $f_{14}$, sum of different powers. GLOBAL fails for the tightest target levels for the functions $f_{10}, f_{11}, f_{13}$, and $f_{14}$, but is the fastest for the loose target levels of $f_{12}$. Overall, it belongs to the fastest algorithms for this class of functions; if it can find the desired target level, it finds it quickly. This can be attributed to its local search procedure, BFGS, which is a fast local search method in itself. Based on the results of the multistart BFGS (Ros, 2009a), which 
P. Pošík, W. Huyer, and L. Pál

Table 7: ERT on $f_{15}-f_{19}$ in 5-D over $\mathrm{ERT}_{\text {best }}$ obtained in BBOB-2009

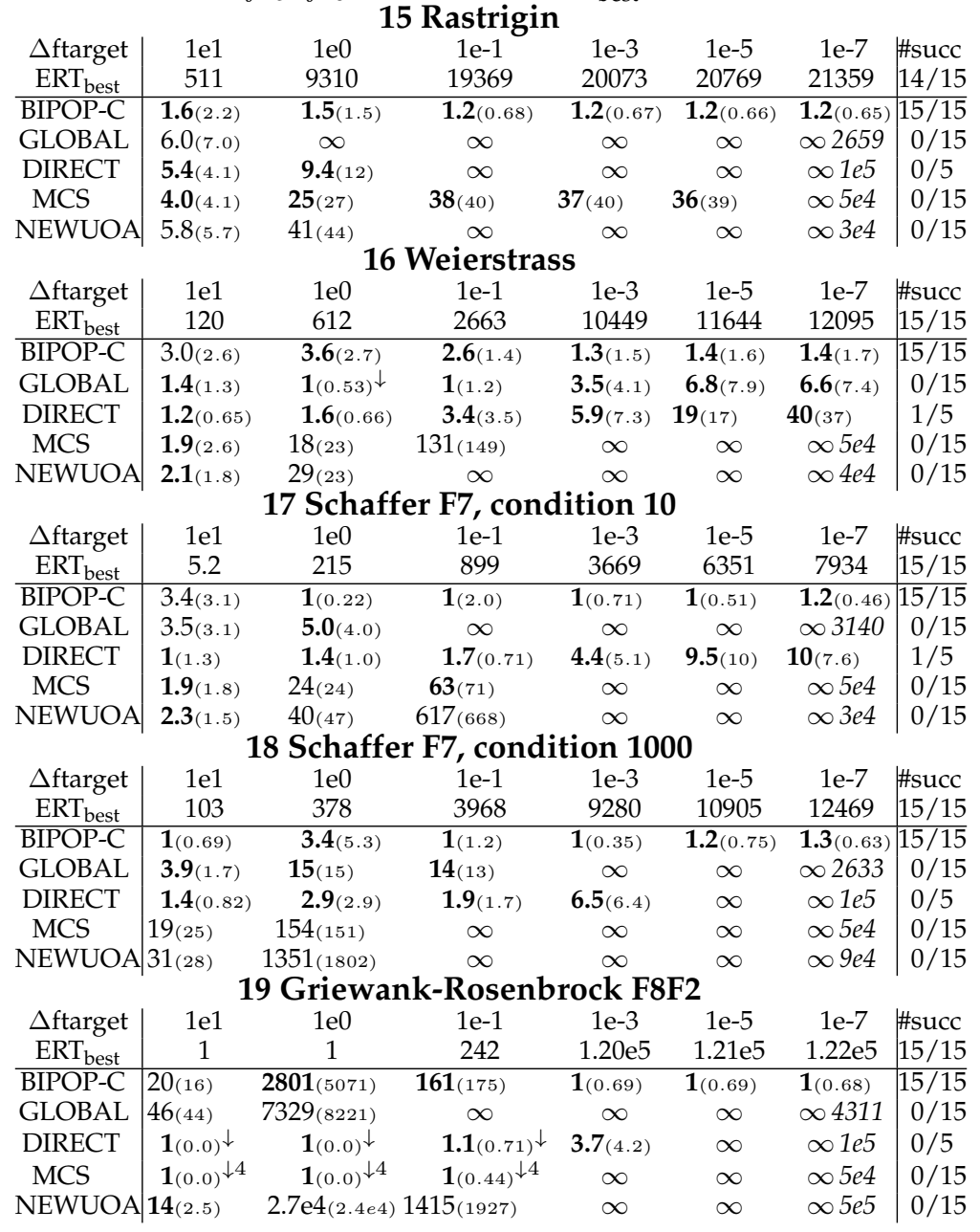

are not shown here, it can be stated that on the moderate and ill-conditioned functions BFGS itself is equally good or better than the GLOBAL algorithm. In both methods, however, some parameters are set differently.

\subsection{Multimodal functions $f_{15}-f_{19}$}

The results for the multimodal functions $f_{15}-f_{19}$ are aggregated in the ECDF graphs of ERT for the 5-D and 20-D functions in Figs. 1 and 2, respectively, in the bottom left part. The detailed results are presented in Table 7 for the 5-D functions, and in Table 8 for the 20-D functions.

In the 5-D space, the performance of all algorithms in the beginning of the search is quite similar. For $100 D<\#$ FEs $<1000 D$, BIPOP-CMA-ES and DIRECT solve the highest proportion of the problems (around 30\%). The performance of the other algorithms starts to decline. Eventually, BIPOP-CMA-ES solves all the problems (needing about $10^{5} \mathrm{D}$ evaluations). DIRECT solved almost $70 \%$ of the problems, MCS and GLOBAL about $40 \%$, and NEWUOA below $30 \%$. 
Table 8: ERT on $f_{15}-f_{19}$ in 20-D over $\mathrm{ERT}_{\text {best }}$ obtained in BBOB-2009

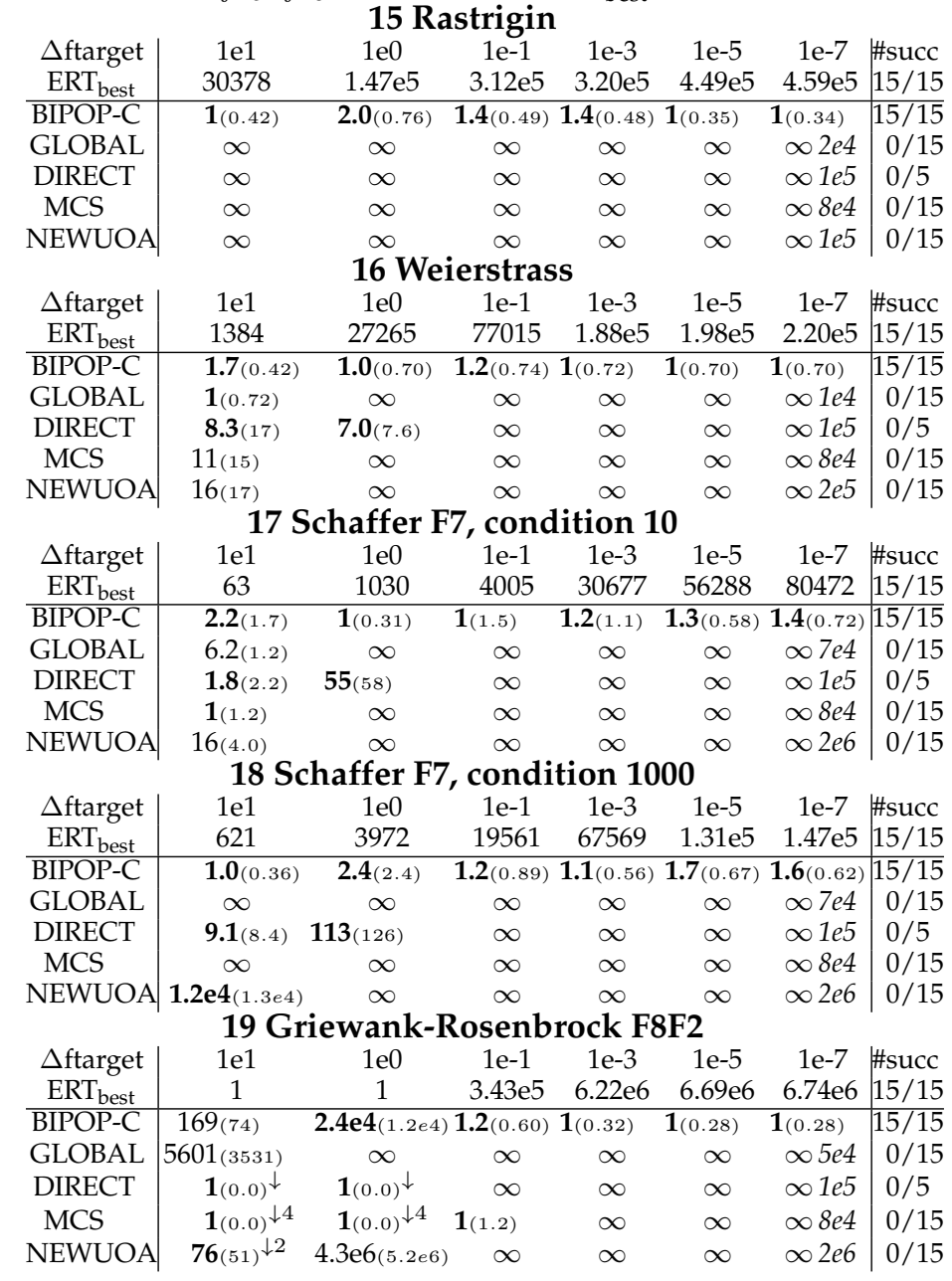

For the 20-D functions, the performance of all algorithms dropped significantly with the exception of BIPOP-CMA-ES. For low evaluation budgets (\#FEs $<100 D$ ), MCS provides the best results (about $10 \%$ ) closely followed by DIRECT. Around $100 D$ evaluations, BIPOP-CMA-ES starts to dominate, eventually solving all the functions, needing $4 \cdot 10^{5} \mathrm{D}$ evaluations. The other algorithms are with a huge gap behind: DIRECT solved about $20 \%$, MCS $15 \%$, NEWUOA $12 \%$, and GLOBAL around $10 \%$.

Regarding the performance of the algorithms for individual functions in this class, the relations are not so obvious. Leaving out the BIPOP-CMA-ES, which solved all the functions, the performance of DIRECT in 5-D is quite surprising. Launching a local search procedure for low-dimensional versions of these functions seems to be only a waste of resources for this class of the problems (see Sec. 7.2 for a supporting argument). For 20-D, DIRECT, MCS, GLOBAL, and NEWUOA are only able to find the loose target levels $\left(>10^{-1}\right)$ which are obviously not very hard to find. The functions in this class have some global structure which can be exploited if the algorithm uses some smoothing mechanism. This is the case for BIPOP-CMA-ES and we conjecture it is the 
P. Pošík, W. Huyer, and L. Pál

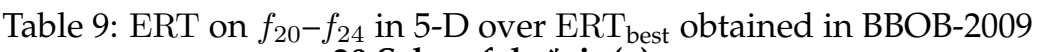

\begin{tabular}{|c|c|c|c|c|c|c|c|}
\hline \multicolumn{8}{|c|}{20 Schwefel $x^{*} \sin (x)$} \\
\hline$\Delta$ ftarget & $1 \mathrm{e} 1$ & $1 \mathrm{e} 0$ & $1 \mathrm{e}-1$ & $1 e-3$ & $1 e-5$ & $1 e-7$ & \#succ \\
\hline $\mathrm{ERT}_{\text {best }}$ & 16 & 851 & 38111 & 54470 & 54861 & 55313 & t/ 13 \\
\hline $\mathrm{BIPOP}-\mathrm{C}$ & $3.3(2.7)$ & $8.2(10)$ & $\mathbf{2 . 8}(1.0)$ & $\mathbf{2 . 1}(0.81)$ & $2.2(0.82)$ & $2.2(0.82)$ & $5 / 15$ \\
\hline GLOBAL & $17(4.9)$ & $18(19)$ & $\infty$ & $\infty$ & $\infty$ & $\infty 2276$ & $0 / 15$ \\
\hline DIRECT & $3.8(0.03)$ & $\mathbf{1 . 5}(5.9 e-4)$ & $\infty$ & $\infty$ & $\infty$ & $\infty 1 e 5$ & $0 / 5$ \\
\hline MCS & 2.7(1.6 & $\mathbf{1}_{(1.8)^{\downarrow 2}}$ & 9.1(10) & 6.4(6.9) & $6.4(7$ & $6.3(7.1)$ & $2 / 15$ \\
\hline \multicolumn{8}{|c|}{21 Gallagher 101 peaks } \\
\hline$\Delta \mathrm{fta}$ & $1 \mathrm{e} 1$ & $1 \mathrm{e} 0$ & $1 e-1$ & $1 e-3$ & $1 e-$ & $1 e-7$ & succ \\
\hline ERT & 41 & 1157 & 1674 & 1705 & 1729 & 1757 & $14 / 15$ \\
\hline$\overline{\mathrm{BIPO}}$ & $2.3(2.1)$ & $14(8.8)$ & $24(35)$ & $25(36)$ & $25(36)$ & $25(36)$ & $15 / 15$ \\
\hline GLO & $2.3(2$ & $\mathbf{1 . 1}(0.87$ & $1(085)$ & $\mathbf{1}(0.83)$ & $\mathbf{1}_{(0 .}$ & $\mathbf{1}(0.81)$ & $14 / 15$ \\
\hline DIR & & & & 2. & & 19 & $4 / 5$ \\
\hline MC & 1.0 & 3.9 & b. & 5. & & & $14 / 15$ \\
\hline NEV & 1.1 & \multicolumn{3}{|c|}{22 Gallagher 21 peaks } & & & $15 / 15$ \\
\hline & $1 \mathrm{e} 1$ & $1 \mathrm{e} 0$ & $1 e-1$ & $1 e-3$ & $1 \mathrm{e}-$ & & suc \\
\hline ER & 71 & 386 & 938 & 1008 & 1040 & 1068 & $4 / 15$ \\
\hline$\overline{\mathrm{BIPO}}$ & $6.9(11)$ & $20_{(14)}$ & $45(94)$ & $42(88)$ & $41(85)$ & $40(83)$ & $15 / 15$ \\
\hline GLC & $3.6(1$. & $1.3(0.90)$ & $\mathbf{1}_{(1.2)}$ & $\mathbf{1}_{(1.1)} \downarrow 2$ & $\downarrow^{2}$ & $\mathbf{1}_{(1.0)^{\downarrow}}{ }^{2}$ & $14 / 15$ \\
\hline DIR & 1 & & $12(11)$ & $22(13)$ & $128(1$ & 400 & $1 / 5$ \\
\hline Mc & $1.0_{(0}$ & 1.1(1.3) & $12(14)$ & $11_{(13)}$ & $111_{(13)}$ & 1 & $14 / 15$ \\
\hline NEW & $2.1(3$ & 2.1 $(2.2)$ & $\begin{array}{l}2.0(3.1)^{\downarrow} \\
\text { Katsuv }\end{array}$ & $\operatorname{lras}^{2.1}(2.8)^{\downarrow}$ & $2.3(2$ & $.4(2.7)$ & $15 / 15$ \\
\hline & $1 \mathrm{e}^{\mathrm{T}}$ & $1 \mathrm{e} 0$ & $1 e-1$ & $1 e-3$ & & & \#succ \\
\hline & 3.0 & 518 & 14249 & 31654 & U & 4256 & $5 / 15$ \\
\hline $\mathrm{BIPC}$ & $1.7_{(1.8)}$ & $13(15)$ & $3.7(4.2)$ & $\mathbf{1 . 8}(1.9)$ & $1.8_{(1}$ & 8) & $15 / 15$ \\
\hline & & 10 & & $\infty$ & $\propto$ & & $0 / 15$ \\
\hline & & & $5.7(7.0)$ & $6.0(6.3)$ & $\alpha$ & & $0 / 5$ \\
\hline & 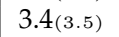 & 3) & $51(57)$ & $\infty$ & $\alpha$ & & $0 / 15$ \\
\hline NEWUOA & $6.2(4.2)$ & $\begin{array}{l}2.4(2.5) \\
24 \mathrm{Lu}\end{array}$ & $7.1(8.1)$ & $\stackrel{\infty}{\text { trigi }}$ & $\infty$ & $3 e 4$ & $0 / 1$ \\
\hline & & $1 \mathrm{e} 0$ & $1 e-1$ & $1 e-3$ & & & \#succ \\
\hline & 1622 & $16 \mathrm{e} 5$ & $6.36 \mathrm{e} 6$ & $9.62 \mathrm{e} 6$ & $28 \mathrm{e} 7$ & $28 \mathrm{e} 7$ & $3 / 15$ \\
\hline & & $6(2.7)$ & $\mathbf{1}_{(0.92)}$ & $\mathbf{1}(1.1)$ & $\boldsymbol{1}_{(1 .}$ & & $3 / 15$ \\
\hline & & $\infty$ & $\propto$ & $\alpha$ & $\infty$ & & $0 / 15$ \\
\hline DIR & & $(2.3)$ & 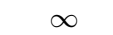 & $\infty$ & $\infty$ & & $0 / 5$ \\
\hline $\mathrm{MC}$ & $7.0_{(8 .}$ & $3.5(3.7)$ & $\propto$ & & $\infty$ & 24 & $0 / 15$ \\
\hline NEWUOA & $\mathbf{2 . 9}(2.2)$ & $2.1(2.2)$ & $\infty$ & $\infty$ & $\infty$ & $\infty 3 e 4$ & $0 / 15$ \\
\hline
\end{tabular}

reason for its superior behavior.

\subsection{Multimodal functions with weak structure $f_{20}-f_{24}$}

The results for the multimodal functions $f_{20}-f_{24}$ are aggregated in the ECDF graphs of ERT for the 5-D and 20-D functions in Figs. 1 and 2, respectively, in the bottom right part. The detailed results are presented in Table 9 for the 5-D functions, and in Tables 10 for the 20-D functions.

For the 5-D functions, until $100 D$ evaluations NEWUOA and MCS are in the lead, DIRECT and BIPOP-CMA-ES have a similar performance, and GLOBAL is the least successful because of its initial random phase. After $100 D$ evaluations, all algorithms solved approx. $20 \%$ of the problems. For $100 D<$ \#FEs $<3 \cdot 10^{3} D$, GLOBAL is the most successful algorithm reaching about $50 \%$ of the solved problems and is very close to the best BBOB-2009 competitor. For budgets larger than $10^{4} D$, BIPOP-CMA-ES leads and eventually reaches $100 \%$ needing at least $10^{6} \mathrm{D}$ evaluations. MCS placed second with $70 \%$ of the solved problems. DIRECT, NEWUOA, and GLOBAL solved 60, 56, 
Table 10: ERT on $f_{20}-f_{24}$ in 20-D over ERT $\mathrm{E}_{\text {best }}$ obtained in BBOB-2009

\begin{tabular}{|c|c|c|c|c|c|c|c|}
\hline \multicolumn{8}{|c|}{20 Schwefel $x^{*} \sin (x)$} \\
\hline$\Delta$ ftarget & $1 \mathrm{e} 1$ & $1 \mathrm{e} 0$ & $1 \mathrm{e}-1$ & $1 e-3$ & $1 e-5$ & $1 \mathrm{e}-7$ & \#suce \\
\hline ERT $_{\text {best }}$ & 82 & 46150 & $3.10 \mathrm{e} 6$ & $5.54 \mathrm{e} 6$ & $5.59 \mathrm{e} 6$ & $5.64 \mathrm{e} 6$ & 7.14 \\
\hline BIPOP-C & $4.3(1.0)$ & 9.2(4.1) & $\mathbf{1}(0.54)$ & $\mathbf{1}(0.34)$ & $\mathbf{1}(0.34)$ & $\mathbf{1}(0.34)$ & $14 / 15$ \\
\hline GLOBAL & $5.2(0.38)$ & $\mathbf{1 . 6}(1.7)^{\downarrow 3}$ & $\infty$ & $\infty$ & $\infty$ & $\infty 8 e 4$ & $0 / 15$ \\
\hline DIRECT & $31(6.1 e-3)$ & $\infty$ & $\infty$ & $\infty$ & $\infty$ & $\infty 1 e 5$ & $0 / 5$ \\
\hline MCS & $4.7(0.51)$ & 12(13) & $\infty$ & $\infty$ & $\infty$ & $\infty 8 e 4$ & $0 / 1$ \\
\hline NEWUOA & $\mathbf{1}(0.45)^{\downarrow 4}$ & $15(18)$ & $\infty$ & $\infty$ & $\infty$ & $\infty 4 e 5$ & $0 / 1$ \\
\hline \multicolumn{8}{|c|}{21 Gallagher 101 peaks } \\
\hline$\Delta$ ftarget & $1 \mathrm{e} 1$ & $1 \mathrm{e} 0$ & $1 e-1$ & $1 e-3$ & $1 e-5$ & $1 e-7$ & łsuc \\
\hline $\mathrm{ERT}_{\text {best }}$ & 561 & 6541 & 14103 & 14643 & 15567 & 17589 & $15 / 1$ \\
\hline BIPOP-C & $3.2(5.5)$ & $55(48)$ & $48(92)$ & $46(89)$ & $43(85)$ & $39(75)$ & $13 / 1$ \\
\hline GLOBAL & $\mathbf{1}_{(0.26)}$ & $\mathbf{1}(1.3)$ & $\mathbf{1}(1.2)$ & $\mathbf{1}(1.1)$ & $\mathbf{1}(1.1)$ & $2.1(2.5)$ & $0 / 15$ \\
\hline DIRECT & $3.3(2.2)$ & $27(33)$ & $\infty$ & $\infty$ & $\infty$ & $\infty 1 e 5$ & $0 / 5$ \\
\hline MCS & $26(36)$ & $32(32)$ & $26(28)$ & $25(28)$ & $23(25)$ & $32(36)$ & $2 / 1$ \\
\hline \multicolumn{8}{|c|}{22 Gallagher 21 peaks } \\
\hline$\Delta \mathrm{fta}$ & $1 \mathrm{e} 1$ & $1 \mathrm{e} 0$ & $1 \mathrm{e}-1$ & $1 \mathrm{e}-3$ & $1 e-$ & $1 \mathrm{e}-7$ & \#succ \\
\hline $\mathrm{ERT}_{\text {best }}$ & 467 & 5580 & 23491 & 24948 & 26847 & $1.35 \mathrm{e} 5$ & $12 / 1$ \\
\hline BIPOP-C & $6.8(13)$ & $13(21)$ & $215(264)$ & $202(247)$ & $188(231)$ & $37(45)$ & $5 / 1$ \\
\hline GLO & $\mathbf{1 . 1}(0.54$ & $\mathbf{1}(1.5)$ & $\mathbf{1}(1.2)$ & $\mathbf{1}(1.1)$ & $\mathbf{1}_{(0.99)}$ & $1.3(1.5)$ & $0 / 15$ \\
\hline DIRECT & $10(10)$ & $16(20)$ & $\infty$ & & $\infty$ & $\infty 1 e 5$ & $0 / 5$ \\
\hline MCS & $17(43)$ & $20(25)$ & $50(53)$ & $47(51)$ & $\infty$ & $\infty 8 e 4$ & $0 / 1$ \\
\hline NEWUOA & $\mathbf{1}(1.2)^{\downarrow}$ & $4.9(6.4)$ & $6.8(7.9)$ & $6.4(7.5)$ & $\mathbf{6 . 0}(6.9)$ & $1.2_{(1.4)}$ & $7 / 1$ \\
\hline \multicolumn{8}{|c|}{23 Katsuuras } \\
\hline$\Delta \mathrm{ft}_{\mathrm{t}}$ & $1 \mathrm{e} 1$ & $1 \mathrm{e} 0$ & $1 e-1$ & $1 e$ & $1 \mathrm{e}$ & $1 \mathrm{e}-7$ & \\
\hline $\mathrm{ERT}_{\text {best }}$ & 3.2 & 1614 & 67457 & $4.89 \mathrm{e} 5$ & $8.11 \mathrm{e} 5$ & $8.38 \mathrm{e} 5$ & $15 / 1$ \\
\hline BIPOP-C & $4.3(4.5)$ & $32(33)$ & $\mathbf{1}(0.83)$ & $2.0(1.5)$ & $1.2(0.88)$ & $1.2(0.85)$ & $15 / 1$ \\
\hline GLOBAL & $2.8(2.7)$ & $\mathbf{1}_{(0.93)^{\downarrow 4}}$ & $\infty$ & $\infty$ & $\infty$ & $\infty 9259$ & $0 / 1$ \\
\hline DIRECT & 4.1(6.7) & $52(73)$ & $\infty$ & $\infty$ & $\infty$ & $\infty 1 e 5$ & $0 / 5$ \\
\hline MCS & $1.3(1.4)$ & $124(131)$ & $\infty$ & $\infty$ & $\infty$ & $\infty 8 e 4$ & $0 / 1$ \\
\hline NEWUOA & $12(8.3)$ & $3.5(3.3)^{\downarrow 3}$ & $32(36)$ & $\infty$ & $\infty$ & $\infty 2 e 5$ & $0 / 1$ \\
\hline \multicolumn{8}{|c|}{24 Lunacek bi-Rastrigin } \\
\hline$\Delta$ ftarget & 1e1 & $1 \mathrm{e} 0$ & $1 e-1$ & $1 e-3$ & $1 e-5$ & $1 \mathrm{e}-7$ & \#succ \\
\hline $\mathrm{ERT}_{\text {best }}$ & $1.34 \mathrm{e} 6$ & $7.48 \mathrm{e} 6$ & $5.19 \mathrm{e} 7$ & $5.20 \mathrm{e} 7$ & $5.20 \mathrm{e} 7$ & $5.20 \mathrm{e} 7$ & $3 / 1$ \\
\hline$\overline{\mathrm{BIPOP}-\mathrm{C}}$ & $\mathbf{1}(0.90)$ & $\mathbf{1}(0.95)$ & $\mathbf{1}(1.0)$ & $\mathbf{1}_{(0.98)}$ & $\mathbf{1}(1.0)$ & $\mathbf{1}(1.0)$ & $3 / 1$ \\
\hline GLOBAL & $\infty$ & $\infty$ & $\infty$ & $\infty$ & $\infty$ & $\infty 3 e 4$ & $0 / 1$ \\
\hline DIRECT & $\infty$ & $\infty$ & $\infty$ & $\infty$ & $\infty$ & $\infty 1 e 5$ & $0 / 5$ \\
\hline MCS & $\infty$ & $\infty$ & $\infty$ & $\infty$ & $\infty$ & $\infty 8 e 4$ & $0 / 1$ \\
\hline NEWUOA & $\infty$ & $\infty$ & $\infty$ & $\infty$ & $\infty$ & $\infty 2 e 5$ & $0 / 1$ \\
\hline
\end{tabular}

and $53 \%$, respectively.

Both Gallagher's functions, $f_{21}$ and $f_{22}$, were solved by all algorithms. For $f_{23}$, Katsuura's, and $f_{24}$, Lunacek bi-Rastrigin, all the algorithms (except BIPOP-CMA-ES) were only able to find loose target levels $>10^{-3}$. MCS beats GLOBAL, DIRECT, and NEWUOA thanks to $f_{20}$, Schwefel's function, which is only solved by BIPOP-CMA-ES and MCS.

In the 20-D space, around $10 D$ evaluations, NEWUOA is the most successful algorithm with $10 \%$ already solved, while the other algorithms are below 5\%. For $100 D<\#$ FEs $<2 \cdot 10^{4} D$, GLOBAL solves the largest proportion of the problems reaching about $50 \%$ and is the best BBOB-2009 algorithm for budgets between $100 \mathrm{D}$ and $2000 D$ evaluations. NEWUOA closely follows GLOBAL. BIPOP-CMA-ES, MCS, and DIRECT have a gap of about 20 to $40 \%$. For \#FEs $>2 \cdot 10^{4} D$, BIPOP-CMA-ES wins solving $100 \%$ of the problems, NEWUOA beats GLOBAL by a few percent both solving around $50 \%$ of the problems. MCS with $40 \%$ is a way in front of DIRECT with only $20 \%$ of success. 
P. Pošík, W. Huyer, and L. Pál

Table 11: The number of functions (out of 24) for which the algorithm found the ultimate precision of $10^{-8}$ for at least 1 run (out of 15) on the function.

\begin{tabular}{lrrrrr}
\hline$D$ & 2 & 3 & 5 & 10 & 20 \\
\hline BIPOP-CMA-ES & 24 & 24 & 23 & 23 & 22 \\
GLOBAL & 18 & 16 & 11 & 8 & 5 \\
DIRECT & 24 & 19 & 9 & 3 & 2 \\
MCS & 19 & 16 & 11 & 8 & 5 \\
NEWUOA & 21 & 18 & 11 & 11 & 11 \\
\hline
\end{tabular}

DIRECT can solve only the loose target level problems for $f_{21}, f_{22}$, and $f_{23}$. MCS solved $f_{21}$, but for $f_{22}$ and $f_{23}$ it only reached the targets $10^{-3}$ and above. GLOBAL and NEWUOA solved very quickly both Gallagher's functions, $f_{21}$ and $f_{22}$. (The shape of the peaks in these functions is very suitable for the local search engine of GLOBAL (BFGS) and for NEWUOA.) They were only partially successful in solving the rest of the functions.

\section{Discussion by Algorithm}

In this section, we look at the results from the point of view of individual algorithms. A global view of the algorithm results is presented in Table 11. The table items describe the number of functions for which we are able to compute a finite ERT, i.e. the number of functions for which at least 1 instance was solved to the ultimate precision $10^{-8}$ by the respective algorithm. Despite the fact that the table shows only one very rough particular view of the results, it can be seen that the BIPOP-CMA-ES scales the best with the dimension. For NEWUOA, we can observe a big drop in the number of solved functions after $D=3$, but then its performance stays rather constant. Another point to note is almost the same profile of MCS and GLOBAL. This is a pure coincidence-there is a substantial proportion of functions where both methods behave quite differently. The DIRECT is relatively successful for low-dimensional functions $(D \leq 3)$, but its success rate then drops significantly.

The following subsections present a detailed discussion of the results broken down by the three main algorithms under the study. The NEWUOA and BIPOP-CMA-ES algorithms are discussed only in the summary discussion of strengths and weaknesses of the individual algorithms in Sec. 7.4.

\subsection{Discussion on DIRECT}

DIRECT's approach to the search is a systematic sampling of points lying in a grid combined with the selection of promising regions to sample. The method, however, uses no dedicated local search algorithm to improve the quality of sampled points. Thus, DIRECT's emphasis on the global search is much larger than that of the other methods.

Due to the absence of the local search algorithm, DIRECT is generally the slowest of the compared methods and within its evaluations budget it often is not able to find the tight target levels. As expected, in higher dimensions both the effectiveness and the efficiency of DIRECT is worse than for small $D$, since the resulting grid is less dense.

DIRECT works on problems where the main trends provide high enough signal to DIRECT's decision process. These are primarily easy functions like $f_{1}$, sphere, and $f_{5}$, linear slope. DIRECT can solve these functions even to tight target levels, but its 
efficiency is not good (it is several orders of magnitude slower than the best BBOB-2009 algorithm).

DIRECT can compete with other algorithms (with the exception of BIPOP-CMAES) on multimodal functions which (1) provide a strong enough signal (have a global structure), and (2) do not offer an advantage to algorithms which use local search to improve the sampled points. In the multimodal functions subgroup $\left(f_{15}-f_{19}\right)$, DIRECT placed second after BIPOP-CMA-ES, leaving MCS and GLOBAL at most $30 \%$ of solved problems behind (for 3-D and 5-D). In 20-D, its gap to the third algorithm is much lower, but it is still second after BIPOP-CMA-ES and about $5 \%$ better than the next competitor. For the subgroup of multimodal functions with weak structure, DIRECT solves a lower number of problems. These functions do not provide so strong signal to DIRECT's decision process. Moreover, the Gallagher's functions $f_{21}$ and $f_{22}$, which were not solved by DIRECT for $D \geq 10$, are known to be efficiently solvable by a restarted local optimizer, so that the other methods (GLOBAL, MCS, NEWUOA) gained their lead over DIRECT mainly on these functions.

\subsection{Discussion on MCS}

MCS1 reached the lowest target function value $f_{\mathrm{t}}^{*}:=f_{\mathrm{opt}}+10^{-8}$ for all instances of the linear slope function $f_{5}$ after $2 D+1$ function calls, i.e., after the initialization procedure, since this function has its minimizer in a corner and the initialization list MCS1 contains the boundary values of all variables. The other problems where all instances, dimensions and precision levels were solved are $f_{1}$ (sphere function, quadratic and separable), $f_{8}$ (Rosenbrock function, original), and $f_{9}$ (Rosenbrock function, rotated). The good performance of MCS on $f_{8}$ and $f_{9}$ is mainly due to the local search algorithm. For all instances and dimensions of $f_{9}$ the value $f_{\mathrm{t}}^{*}$ was already achieved by the first call to the local search algorithm and the same is true for $84 \%$ of the instances and dimensions of $f_{8}$; in the remaining cases $f_{\mathrm{t}}^{*}$ was reached by the second call to the local search.

MCS performs well on the separable functions $\left(f_{1}-f_{5}\right)$ and the functions with low or moderate condition $\left(f_{6}-f_{9}\right)$ at least for small dimensions, and the same holds for the ill-conditioned function $f_{12}$ and the multimodal functions $f_{15}$ and $f_{19}-f_{22}$. The value $f_{\mathrm{t}}^{*}$ was reached for at least 12 (out of 15) instances in 2-D for $f_{4}$ and $f_{6}$, up to 3-D for $f_{3}$, $f_{7}, f_{15}, f_{19}$, and $f_{20}$, and up to 5-D for $f_{2}, f_{21}$, and $f_{22}$; in addition, it was reached for 8 instances of $f_{22}$ for 10-D. For all other dimensions $f_{\mathrm{t}}^{*}$ was only reached in a minority of the instances for these functions. The only function apart from the easy functions where reaching $f_{\mathrm{t}}^{*}$ occurred for at least 2 instances of each dimension is Gallagher's Gaussian 101-me peaks function $f_{21}$ (101 local optima, moderate conditioning around the global optimum). In $63 \%$ of the "successful" cases for $f_{2}-f_{4}, f_{6}, f_{7}, f_{12}, f_{15}$, and $f_{19}-f_{22}$, the value $f_{\mathrm{t}}^{*}$ was already achieved by MCS1, in $15 \%$ by MCS2, and in $11 \%$ each by both MCS3 and later calls to MCS.

The functions for which MCS did not reach $f_{\mathrm{t}}^{*}$ even in low dimensions in a majority of the instances belong to two classes. The functions $f_{10}, f_{11}, f_{13}$, and $f_{14}$ are unimodal with high conditioning, which means that approaching the minimizer becomes more difficult close to the minimizer, and the problem becomes worse in higher dimensions. In low dimensions we may still achieve the precision level $10^{-5}$ or even $10^{-7}$, but in large dimensions $\Delta f_{\text {best }}$ becomes much larger. This is a problem of the local optimization part of MCS; in most cases the local optimization algorithm becomes so slow that the limit $n f_{\max }$ on the number of function calls is reached before a stopping criterion for the local search algorithm is satisfied, and in some cases the local optimization stops due to not improving the function value any more before reaching a local minimizer. 
The second class of those functions are the multimodal functions $f_{16}$ (highly rugged), $f_{17}$ (highly multimodal), $f_{18}$ (moderately ill-conditioned counterpart of $f_{17}$ ), $f_{23}$ (highly rugged, more than $10^{D}$ global optima), and $f_{24}$ (highly multimodal, funnel of one non-global minimizer has roughly $70 \%$ of the search space volume); $f_{24}$ in 2-D is the only exception since $f_{\mathrm{t}}^{*}$ was reached for 8 instances. In many of the runs where the final precision was not achieved (particularly the ones with higher dimensions, but also some of the unsuccessful runs for $f_{24}$ for 2-D), the algorithm was repeatedly caught in the same non-global minimum, and the final $f_{\text {best }}$ was larger in higher dimensions. The deterministic nature of MCS does not allow to recover from getting stuck when the algorithm happened to rate the region containing the global minimum as unpromising.

In order to study the effect of the local searches on the performance of the algorithm, we also applied MCS1 with $n s_{\text {loc }}=0$ (no local search), $s_{\max }=25 D$ (a higher value than before to compensate for the lack of a local optimizer), and $n f_{\max }=100000$ (the budget used for DIRECT) to the test functions, i.e., no restarts were made and the whole (larger) function evaluation budget was used for a single call to MCS. Since no random element is contained, each problem was only treated once. Most of the results for the "easy" functions $f_{1}-f_{4}, f_{8}, f_{9}, f_{12}$, and $f_{19}-f_{22}$ are far inferior, which suggests that the local searches are to a great extent responsible for the good results. For the functions $f_{16}(D \leq 10), f_{17}(D=5,10)$, and $f_{18}(D=2,3,5)$, however, tighter precision levels were reached, and the improvement is largest for $f_{16}$ for $D=2,3,5$. The results support the conjecture made in Sec. 6.5 that launching a local search procedure is only a waste of resources for the 5-D problems $f_{15}-f_{19}$ for three of these problems.

In Huyer and Neumaier (1999) it was already observed that the performance of MCS was not satisfactory for most of the problems with dimensions $D \geq 10$ and for hard multimodal test problems, but except for the two-dimensional Rosenbrock function, the test problems considered there were different from the BBOB test set. Moreover, only a single target function value was considered in that paper. Failures of MCS due to shortcomings of the local search algorithm were not encountered by the authors before.

\subsection{Discussion on GLOBAL}

GLOBAL obtains the highest number of successful trials for functions from the separable, moderate, ill-conditioned and weak-structure subgroups, specifically for $f_{1}, f_{2}, f_{5}$, $f_{6}, f_{8}, f_{9}, f_{10}, f_{11}, f_{12}, f_{21}$ and $f_{22}$ in dimensions 2,3 , and 5. For $f_{1}, f_{2}, f_{5}, f_{8}$ and $f_{9}$, GLOBAL obtained successful trials for all dimensions.

Considering the different function subgroups, the best behavior of GLOBAL can be observed on the separable (except $f_{3}$ and $f_{4}$ ), moderately conditioned (except $f_{7}$ ) and on ill-conditioned functions subgroups. These functions are unimodal (or have a quite large attraction region of the global optimum, $f_{8}$ and $f_{9}$ ), which is the main reason for the good performance of GLOBAL. The results on the attractive sector function $f_{6}$ in 20-D can be improved by increasing the function evaluation limit of the BFGS method. Finding the best solution for $f_{8}$ and $f_{9}$ is mainly due to the BFGS local search and partly to the property of these functions presented previously. For the discus function $f_{11}$, GLOBAL cannot find a target precision value better than $10^{-1}$ in $20-\mathrm{D}$ due to a problem of the BFGS local search. In this case the method stops too early because it cannot decrease the objective function along the current search direction. GLOBAL also performs well on the Gallagher's multimodal functions $f_{21}$ and $f_{22}$ with weak global structure, thanks to restarting the local search method.

The hardest problems, for which the method did not reach the solution in higher 
dimensions, are the multimodal Rastrigin functions $f_{3}, f_{4}, f_{15}$ and $f_{24}$. In case of the $f_{24}$ function, even in 2-D we cannot find a better target precision value than $10^{-2}$, while in the case of the $f_{3}, f_{4}, f_{15}$ functions the $\Delta f_{\text {best }}$ value is not better than 10 in 5 -D and $10^{2}$ in $20-\mathrm{D}$, respectively. The common property of these functions is that they have more than $10^{D}$ local optima. Therefore the algorithm cannot discover the overall function structure. Moreover, the size of the region of attraction of the global optimum is small for these problems, and hence the algorithm fails to satisfactorily sample in these regions. GLOBAL also fails to reach precision levels better than 1 on the $f_{17}$ and $f_{19}$ multimodal functions with adequate global structure in 5-D and 10 in 20-D. The reason is the same as presented above.

\subsection{Summary of the Strengths and Weaknesses}

The DIRECT algorithm is competitive only for the low-dimensional functions and the problems with loose target levels. From the ECDF graphs of ERT, it is obvious that the results for DIRECT are virtually never part of the Pareto front, i.e. DIRECT is almost always dominated by some other algorithm(s).

The most obvious strength of the MCS algorithm is its initial phase. In all reported dimensions, for budgets lower than $100 D$, it belongs to the 2 best performing algorithms across all function groups. MCS also exhibits competitive performance on separable functions. The weakness of this algorithm is the employed local search procedure which does not perform well in certain situations, especially for high-conditioned and rugged functions. Moreover, getting stuck in a nonglobal minimizer is a problem occurring for difficult multimodal functions, and, like DIRECT, the splitting procedure handles high dimensions less satisfactorily.

The strength of the GLOBAL algorithm is its ability to reduce the number of calls to the local search procedure. Otherwise, it builds on the strengths of its local search procedures, the Nelder-Mead simplex search and the quasi-Newton method with the BFGS update. Across all function groups and all tested dimensions $(D \leq 20)$, it exhibits the fastest progress between $100 \mathrm{D}$ and $1000 \mathrm{D}$ function evaluations. It works especially well for the class of unimodal ill-conditioned functions. The weakness of GLOBAL is the fact that the first local search is executed only after 300 function evaluations. This deteriorates the performance of GLOBAL in the initial phase. Another weakness of GLOBAL is the sampling phase especially in the case of functions with small basins of attraction, where the algorithm fails to satisfactorily sample in these regions.

The NEWUOA algorithm competes with MCS for the fastest progress in the initial stages of the search (\#FEs $<100 D)$. With increasing dimension, the effectiveness of NEWUOA drops more slowly than for DIRECT, MCS, and GLOBAL, so that it eventually solves the second highest proportion of functions after BIPOP-CMA-ES. The weakness of NEWUOA is its slower rate (compared e.g. to BFGS) with which it updates its quadratic model in the middle stage of the search. This can be the reason for the failure of the method on multimodal functions with an adequate structure.

The most striking advantage of the BIPOP-CMA-ES algorithm is its ability to solve a very large proportion of problems. Its weakness would then be a slower progress in the initial stages of the search. But even in these cases, to solve the same proportion of problems, BIPOP-CMA-ES is usually at most 10 times slower than the fastest of the other algorithms. After about $1000 D$ evaluations, BIPOP-CMA-ES becomes the most successful algorithm among those compared. 


\section{Summary and Conclusions}

In this article, we experimentally compared 5 optimization algorithms and discussed their results, strengths and weaknesses. Their evaluation is related to the particular instances of the individual algorithms in terms of parameter settings and choice of individual components. It is possible that with a different parametrization of the algorithms we could get better results. However, the algorithms were tuned to provide acceptable performance across the test suite, and we believe that the parameter settings are good enough to show the most important aspects of the algorithms.

\subsection{Which Algorithm Should I Choose?}

The results confirmed that there is no best algorithm when compared over all functions, dimensions and target criteria. Despite of that, we can give the following suggestions for the black-box scenario. (If the experimenter knows some characteristics of her problem, we refer her to the respective part of Sec. 6). The suggestions hold across the tested dimensions (with a few exceptions for 2-D and 3-D).

Low evaluations budget, \#FEs $<100 \mathrm{D}$. The mathematical programming algorithms were designed to provide quick progress in the initial stages of the search, and they fulfill this goal. In the initial stages, MCS and NEWUOA usually provide the best results. There is also a substantial proportion of functions which can be solved quickly to a high precision by these solvers.

Intermediate evaluations budget, $100 \mathrm{D}<\mathrm{\# FEs}<1000 \mathrm{D}$. In the middle stage of the search, restarting a good local optimizer can be very rewarding. At these stages, GLOBAL and restarted NEWUOA often belong to the best algorithms.

There are also problems which are very hard for these algorithms and which were solved only by BIPOP-CMA-ES. The other methods are not able to solve these problems in a reasonable time; they need a high number of function evaluations and are stopped too early to solve them. Some form of aggregation or averaging is often needed to solve some of the multimodal benchmark problems, and BIPOP-CMA-ES is the only algorithm in this comparison that performs such aggregation.

The final proportion of solved problems. BIPOP-CMA-ES is a clear winner from this point of view (it usually overtakes the other tested algorithms for budgets larger than \#FEs > 1000D). With NEWUOA and GLOBAL, which placed second and third, it belongs to the class of algorithms which restart an efficient local optimizer (possibly varying some algorithm parameters). On the contrary, MCS and DIRECTrepresentatives of the "divide and conquer" approach-are worse and slower in the later stages of the search.

Despite the fact that the mathematical programming methods often provide some important theoretical properties (convergence properties for DIRECT and MCS; finite number of local searches for GLOBAL), a good stochastic optimizer with origins in evolutionary computation, BIPOP-CMA-ES, is able to (1) find the optimum of a broader class of functions, (2) solve problems with a higher precision, and (3) solve some problems faster.

\subsection{Suggestions for Hybridization}

The evolutionary community can find inspiration in many features of the mathematical programming methods. We can name at least a few.

The successful sampling process of MCS or NEWUOA in the beginning of the search can be used for the population initialization. It may bias the algorithm on the one hand, but could provide an initial boost on the other hand. 
To prevent premature convergence, many evolutionary algorithms (EAs) occasionally incorporate randomly generated individuals into the population (or reinitialize a part of the population). The systematic sampling procedure of DIRECT can be used as a generator of the new population members. It would ensure that the search space is covered, yet the new members would not be mere random guesses, but samples of promising regions.

Many memetic algorithms can be viewed as multistart methods with an EA serving as the generator of the starting points for the local search method. We can use the clustering method of GLOBAL to filter the starting point candidates generated by an EA to reduce the chance of finding an already known local optimum. A different method with the same goal was introduced in the context of genetic algorithms as Clustered Genetic Search (Schaefer, 2007).

It is also possible to use some particular principles of the MP methods to improve the behavior of EAs. One attempt in this direction was already presented in the bachelor thesis of Körner (2011). The principle of DIRECT to select the potentially optimal boxes was used to pre-select the suitable pairs of parents for arithmetic crossover in an otherwise canonical EA. The unpromising pairs are filtered out and more effort is put to crossing over the pairs of parents with high chance of producing a good offspring. Such a modification significantly improved the convergence speed in the beginning of the search.

Acknowledgements. The authors would like to thank to N. Hansen, R. Ros, and A. Auger for the tremendous work during the development of the COCO framework. Many thanks go to A. Neumaier for his patient discussions on global optimization. The first author was supported by the Ministry of Education, Youth and Sport of the Czech Republic with the grant No. MSM6840770012 entitled "Transdisciplinary Research in Biomedical Engineering II". The third author was supported by the grant TÁMOP4.2.2/08/1/2008-0008 of the Hungarian National Development Agency. The authors wish to thank the anonymous reviewers for their useful constructive comments.

\section{References}

Boender, C. G. E., Rinnooy Kan, A. H. G., Timmer, G. T., and Stougie, L. (1982). A stochastic method for global optimization. Mathematical Programming, 22:125-140.

Csendes, T. (1988). Nonlinear parameter estimation by global optimization-efficiency and reliability. Acta Cybernetica, 8(4):361-370.

Csendes, T., Pál, L., Sendin, J. O. H., and Banga, J. R. (2008). The GLOBAL Optimization Method Revisited. Optimization Letters, 2:445-454.

Finck, S., Hansen, N., Ros, R., and Auger, A. (2009a). Real-parameter black-box optimization benchmarking 2009: Presentation of the noiseless functions. Technical Report 2009/20, Research Center PPE.

Finck, S., Hansen, N., Ros, R., and Auger, A. (2009b). Real-parameter black-box optimization benchmarking 2009: Presentation of the noiseless functions. Technical Report 2009/20, Research Center PPE. Updated February 2010.

Finkel, D. E. (2003). DIRECT Optimization Algorithm User Guide.

Hansen, N. (2009). Benchmarking a BI-population CMA-ES on the BBOB-2009 function testbed. In Rothlauf (2009), pages 2389-2396.

Hansen, N., Auger, A., Finck, S., and Ros, R. (2009a). Real-parameter black-box optimization benchmarking 2009: Experimental setup. Technical Report RR-6828, INRIA. 
P. Pošík, W. Huyer, and L. Pál

Hansen, N., Auger, A., Ros, R., Finck, S., and Pošík, P. (2010). Comparing results of 31 algorithms from the black-box optimization benchmarking BBOB-2009. In Proceedings of the 12th annual conference companion on Genetic and evolutionary computation, GECCO '10, pages 16891696, New York, NY, USA. ACM.

Hansen, N., Finck, S., Ros, R., and Auger, A. (2009b). Real-parameter black-box optimization benchmarking 2009: Noiseless functions definitions. Technical Report RR-6829, INRIA.

Hoos, H. and Stützle, T. (1998). Evaluating Las Vegas algorithms-pitfalls and remedies. In Proceedings of the Fourteenth Conference on Uncertainty in Artificial Intelligence (UAI-98), pages 238-245.

Huyer, W. and Neumaier, A. (1999). Global optimization by multilevel coordinate search. Journal of Global Optimization, 14(4):331-355-355.

Huyer, W. and Neumaier, A. (2009). Benchmarking of MCS on the noiseless function testbed. http://www.mat.univie.ac.at/ neum/papers.html. P. 989.

Jones, D. R., Perttunen, C. D., and Stuckman, B. E. (1993). Lipschitzian optimization without the Lipschitz constant. Journal of Optimization Theory and Applications, 79(1):157-181.

Kelley, C. T. (1999). Iterative Methods for Optimization. SIAM in Philadelphia.

Körner, P. (2011). Evolutionary algorithms with crossover success estimation. Bachelor thesis, Czech Technical University in Prague.

Nelder, J. and Mead, R. (1965). A simplex method for function minimization. The Computer Journal, 7(4):308-313.

Neumaier, A. (2004). Complete search in continuous global optimization and constraint satisfaction. Acta Numerica, 13:271-369.

Pál, L., Csendes, T., Markót, M. C., and Neumaier, A. (2009). BBObenchmarking of the GLOBAL method for the noiseless function testbed. http://www.mat. univie.ac.at/ neum/papers.html. P. 986.

Pošík, P. (2009). BBOB-benchmarking the DIRECT global optimization algorithm. In Rothlauf (2009), pages 2315-2320.

Powell, M. J. D. (2006). The NEWUOA software for unconstrained optimization without derivatives. Large Scale Nonlinear Optimization, pages 255-297.

Price, K. (1997). Differential evolution vs. the functions of the second ICEO. In Proceedings of the IEEE International Congress on Evolutionary Computation, pages 153-157.

Rinnooy Kan, A. H. G. and Timmer, G. T. (1987). Stochastic global optimization methods Part I: Clustering methods. Mathematical Programming, 39:27-56.

Ros, R. (2009a). Benchmarking the BFGS algorithm on the BBOB-2009 function testbed. In Rothlauf (2009), pages 2409-2414.

Ros, R. (2009b). Benchmarking the NEWUOA on the BBOB-2009 function testbed. In Rothlauf (2009), pages 2421-2428.

Rothlauf, F., editor (2009). Genetic and Evolutionary Computation Conference, GECCO 2009, Proceedings, Montreal, Québec, Canada, July 8-12, 2009, Companion Material. ACM.

Schaefer, R. (2007). Foundations of Global Genetic Optimization, volume 74 of Studies in Computational Intelligence. Springer. 